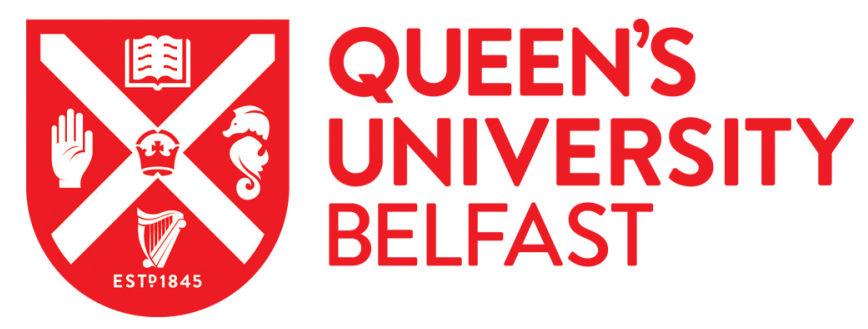

\title{
Adaptive neural integral sliding-mode control for tracking control of fully actuated uncertain surface vessels
}

Van, M. (2019). Adaptive neural integral sliding-mode control for tracking control of fully actuated uncertain surface vessels. International journal of robust and nonlinear control, 29(5), 1537-1557.

https://doi.org/10.1002/rnc.4455

\section{Published in:}

International journal of robust and nonlinear control

\section{Document Version:}

Peer reviewed version

Queen's University Belfast - Research Portal:

Link to publication record in Queen's University Belfast Research Portal

\section{Publisher rights}

(C) 2019 John Wiley \& Sons, Ltd

This work is made available online in accordance with the publisher's policies. Please refer to any applicable terms of use of the publisher.

\section{General rights}

Copyright for the publications made accessible via the Queen's University Belfast Research Portal is retained by the author(s) and / or other copyright owners and it is a condition of accessing these publications that users recognise and abide by the legal requirements associated with these rights.

Take down policy

The Research Portal is Queen's institutional repository that provides access to Queen's research output. Every effort has been made to ensure that content in the Research Portal does not infringe any person's rights, or applicable UK laws. If you discover content in the Research Portal that you believe breaches copyright or violates any law, please contact openaccess@qub.ac.uk. 


\title{
Adaptive Neural Integral Sliding Mode Control for Tracking Control of Fully Actuated Uncertain Surface Vessels
}

\author{
Mien $\operatorname{Van}^{a *}$ \\ ${ }^{a}$ College of Engineering, Mathematics and Physical Sciences, University of Exeter, United Kingdom \\ *Corresponding Author's E-mail: m.van@exeter.ac.uk
}

\begin{abstract}
This paper develops a novel adaptive neural integral sliding mode control (ANISMC) to enhance the tracking performance of fully actuated uncertain surface vessels. The proposed method is built based on an integrating between the benefits of the approximation capability of neural network (NN) and the high robustness and precision of the integral sliding mode control (ISMC). In this paper, the design of NN, which is used to approximate the unknown dynamics, is simplified such that just only one simple adaptive rule is needed. The ISMC, which can eliminate the reaching phase and offer higher tracking performance compared to the conventional sliding mode control, is designed such that the system robust against the approximation error and stabilize the whole system. The design procedure of the proposed controller is constructed according to the backstepping control technique so that the stability of the closed-loop system is guaranteed based on Lyapunov criteria. The proposed method is then tested on a simulated vessel system using computer simulation and compared with other state-of-the-art methods. The comparison results demonstrate the superior performance of the proposed approach.
\end{abstract}

Keywords: Integral sliding mode control, neural network, backstepping control technique, adaptive control, surface vessels.

\section{Introduction}

Marine Vessels have been widely utilized in the sea for several major tasks such as transportation, ocean exploration, oil harvesting, etc. [1-2]. Since the vessels always work in the unstructured and extreme environment, the tracking performance is significantly decreased due to the effects of unmodeled dynamics and 
external disturbances. Therefore, enhancing tracking control of surface vessels, particularly in the presence of model uncertainties and external disturbances, has been significant and attracted a lot of attention from both industrial and academia.

Several control methods have been developed for uncertain surface vessels. Initially, model-free control methods based on PID or PD controllers have been developed [3-4]. Intelligent control methods based on fuzzy logic have also been developed for improving tracking performance [5-6]. However, the employed of the modelfree control methods provide low precision when the system is immersed by high uncertainty and disturbance. In addition, the approaches based on fuzzy logic provides high steady-state error. In order to get higher tracking performance of the system, model-based control methods have been developed. Nevertheless, the performance of the model-based control methods depends hugely on the exact mathematical model of the system, which is difficult to be obtained in practical engineering applications. In the early model based control approach, some control methods such as backstepping control [7] and model predictive control [8-9] have been developed based on the assumption that the accurate model of the system can be obtained. However, when model uncertainty and disturbance are present, these controllers provide big offset error. Due to the necessity of the exact mathematical model of the system in the design of controller, some efforts have been spent to obtain the accurate model. In [10], a dynamic model of the vessel has been built by collecting the data from several experiments, and an adaptive control method has been developed based on the identified model. However, this model identification approach is a tedious process and the accurate of the dynamics model depends on the quality and quantity of the collected data. In addition, due to the limitation of the sensors used to collect the training data, the exact dynamic model of the system is almost impossible to get, particularly for the vessels, which are always working in the unstructured and extreme environment. Therefore, the control system for surface vessels must have capability to robust against the effects of the model uncertainty and disturbance. To obtain this aim, some optimal control methods have been developed to deal with the uncertainty [11-12]. However, the optimization algorithms used in the optimal control usually require high computation. To estimate the model uncertainty in the system, disturbance observer-based controller has been developed [13-14]. The design of this approach includes two stages. In the first stage, a disturbance observer is designed to estimate the uncertainty in the system dynamics. Then, the estimated uncertainty is fed back into the controller to compensate for the uncertainty. Due to the twostage operation, the stability of the closed-loop system may meet difficult to be guaranteed.

In the control engineering's point of view, two approaches could effectively compensate for the effects of the 
uncertainty and disturbance: (i) robust control technique, and (ii) learning technique. In a progress working toward the first approach, several robust control techniques have been developed for the tracking control of surface vessels [15-16]. For example, in [15], a robust control scheme has been built based on a combining between the backstepping control technique and a disturbance observer. Even though the system can guarantee the prescribed performance of the system, the designed controller provided weak robustness against the uncertainties and disturbance. In [16], a distributed consensus robust controller for a group of autonomous underactuated surface vessels has been developed. Sliding mode control (SMC) [17], which is a well-known robust control technique, has been widely developed for surface vessels to robust against the effects of the uncertainties and disturbance [18-19]. Generally, the operation of the SMC consists of two phases: reaching phase and sliding phase. In the reaching phase, the system is forced to reach the sliding surface. In the sliding phase, the system is kept to always maintain on it. However, the presence of the reaching phase could decrease the effectiveness of the SMC [20].

In order to overcome the limitation of the conventional SMC, integral sliding mode control (ISMC) has been developed [21-23]. Particularly, in [21], a design and analysis of the ISMC for fault tolerant control (FTC) of the linear systems has been developed. In [22], a review of the ISMC and its advance techniques has been done. In [23], a nonlinear integral type sliding surface has been developed for both matched and unmatched uncertain systems. Compared to the conventional SMC, the ISMC would offer three major advantages, as has been pointed out in [24]. First, the system eliminates the reaching phase. This property is particularly helpful for practical applications since it helps to guarantee the physical constraints of the system from the initial position. Second, the effects of the matched uncertainty and external disturbance can be suppressed completely and the amplification of the unmatched disturbance will be avoided. Third, the nominal system (the system without uncertainty and disturbance) decides the behavior of the states of matched disturbance system. However, compared to the conventional SMC, according to the author's experience, the design of the ISMC also meets some drawbacks/difficulties such as: (i) it is difficult to select the appropriate sliding surface to get higher performance, (ii) it meets challenging to reconstruct the nominal controller of the system such that the desired response of the system can be satisfied. Because of the drawbacks, the ISMC has been widely applying for the linear systems, where the nominal controller can be well defined, rather than for nonlinear systems [21-24]. Hence, although the ISMC possesses many nice features over the conventional SMC, however, according to the best of the author's knowledge, there are no approaches in the literature to employ this advanced control technique 
for tracking control of surface vessels. This is because the surface vessel is a high nonlinear system, and therefore it may meet difficult to select the appropriate sliding surface and effectively reconstruct the nominal controller and switching term for the system to reject the uncertainties and disturbance. Hence, more efforts should be spent to push forward this interested control technique for surface vessels.

It should be noted that, like the conventional SMC, the ISMC also provides big chattering due to the use of sign function in the design. In order to eliminate the chattering, several approaches have been developed in the literature. The most popular method is to employ the boundary method [21]. This method, however, causes high steady state error due to the effects of the boundary. An alternative method is to employ the disturbance observer (DO) [25]. The basic idea of this approach is to estimate the uncertainty component using a DO, and the estimated uncertainty is then used to compensate for the effects of the uncertainty in the system using feedforward control technique. Since the effects of the uncertainty and disturbance can be mostly compensated by the DO, the sliding gain of the SMC just needs to be selected as a smaller value, which will significantly reduce the chattering. Recently, a new method called conditional integral (CI) has been developed to reduce the chattering [26-27]. In [28], a fuzzy logic inference mechanism has been developed to replace the sign function in the conventional SMC to reduce the chattering. However, this approach reduces the robustness of the SMC. Another method to reduce the chattering is to employ high-order sliding mode control (HOSMC) techniques [29].

In the second approach, due to the approximation capability, learning techniques based on neural network (NN) or fuzzy logic have been widely used to approximate the unknown component in the system [30-31]. The approximation ability has also been applied to approximate the uncertainty of the surface vessels [32-33]. The benefits of this approach are that the unknown dynamic model of the system can be approximated and compensated online. However, the learning process of NN generates high computational burden in the system. Therefore, in order to effectively employ NN into practical applications, the learning mechanism should be simplified. Due to this demand, some efforts have been spent to simply the learning mechanism of NN using the Young's inequality [34-35]. In addition, the learning technique based on NN/fuzzy logic provides certain approximation error. This error depends on many factors of the system such as the structure of the selected $\mathrm{NN} /$ fuzzy logic, the variation of the unknown function, etc. Therefore, appropriate compensation algorithms are needed to compensate for the NN's approximation error.

Due to the advantages of the SMC and NN approximation, several approaches have been developed to combine the benefits of them [36-39]. Lately, for the sake of control design procedure, backstepping control technique has 
been employed into the design of the conventional SMC [40-42] or the hybrid SMC and NN/fuzzy logic [43, 44]. In these hybrid approaches, the NN is used to approximate the unknown dynamics model of the system. Then, the approximated $\mathrm{NN}$ is used to compensate for the effects of the uncertainty, and the SMC is lastly used to compensate for the NN's approximation error and stabilize the whole system. The backstepping control technique is employed to facilitate the design procedure. In this way, the chattering of the SMC can be significantly reduced like a manner to the DO approach. Although the hybrid control approaches based on the conventional NN and conventional SMC would enhance the tracking performance of the system, however, as the aforementioned drawbacks of the conventional SMC and NN, the existing hybrid systems provided two major drawbacks: (1) the system required the reaching phase like the conventional SMC, and (2) the computational burden of the NN was high. From the benefits of the ISMC and NN, it would be desired to develop a hybrid system that combines the benefits of both. Unfortunately, in the literature, very few efforts have been spent to create this important strategy. One may claim that it is quite straightforward to employ the ISMC to replace the conventional SMC in the hybrid SMC and NN. However, as aforementioned discussion, this design step has not been straightforward really. Many issues need to be further addressed when employing ISMC into such hybrid system, such as how to reconstruct the appropriate integral sliding surface taking the benefits of the adaptive NN into account?, and how to design effective nominal controller when integrating the adaptive $N N$ into the system?. According to the best of the author's knowledge, there are no existing answers in the literature for the two given questions. Therefore, further efforts need to be spent to push forward this important progress in the control development. This is one of the most motivations of this paper.

In this paper, a new approach based on an integrating between ISMC and NN is developed for tracking control of surface vessels. First, an integral sliding mode control is studied. The advantages and disadvantages of this controller are discussed. Then, in order to enhance the tracking performance of the system, a novel integral sliding surface based on an adaptive NN is proposed, in which the weight's updating law is simplified using the Young's inequality. In addition, the adaptive NN is designed based on the backstepping strategy so that the stability of the system can be guaranteed based on the Lyapunov stability method. The proposed method is then tested for the simulated surface vessels based on computer simulation and compared with other advanced control techniques. The simulation results confirm the superior performance of the proposed strategy.

In summary, compared with the existing approaches, the novelties of this approach are as follows:

- As far as we know, there are no approaches for the control of surface vessels with the integral sliding 
mode control method. In addition, there are no approaches in the literature to develop hybrid control method based on integral sliding mode control and adaptive NN. The goal of this paper is to fill in the gap in this interesting field.

- A novel integral sliding surface based on an adaptive $\mathrm{NN}$ is proposed. This novelty is very significant in comparing with many other existing approaches. For example, compared to the conventional ISMC [21-24], the proposed method possesses the advantages of the adaptive NN, and thus the chattering is much reduced and no requirement on the bounded value of the uncertainties and disturbance is needed. Compared to the conventional adaptive fuzzy logic/NN approaches [32-35], the proposed approach possesses higher robustness since it preserves the high robustness property of the ISMC. Compared to the hybrid SMC and NN [36-39] or hybrid backstepping, SMC and NN [42-44], the proposed approach offers two major advantages: (i) it eliminates the reaching phase and obtains higher tracking precision due to the property of the ISMC, and (ii) the computational load of the system is lower due to the simple adaptation law of the proposed adaptive NN.

- Based on the backstepping control framework, the control design procedure can be performed easier and the stability of the whole system can be guaranteed based on Lyapunov criteria. Although the backstepping control is a well-known technique in the literature, its employment into ISMC and particularly into the hybrid ISMC and NN is relatively new.

- A comparison between the proposed method with other state-of-the-art methods such as SMC, adaptive backstepping SMC, adaptive NN, and hybrid adaptive NN and SMC (ANSMC) has been performed to verify the effectiveness of the design procedure.

The rest of the paper is organized as follows. Section 2 formulates the problem. Section 3 describes the initial idea of the integral sliding mode control for tracking control of surface vessels. The proposed method based on adaptive neural integral sliding mode control is presented in section 4. Computer simulation for the tracking control of surface vessels and results are discussed in section 5. Section 6 provides conclusions.

\section{Problem Formulation}

Consider the vessels in horizontal motion (only three motion components including surge, sway and yaw are taken into consideration) with the reference coordinate frames illustrated in Fig. 1, where $O_{e} X_{e} Y_{e}$ and $O_{b} X_{b} Y_{b}$ denotes the earth frame and the body-fixed frame, respectively. The origin $O_{e}$ is the original position of the 
desired position, and the origin $O_{b}$ is considered as the center point of the vessel's geometric structure. Based on the defined coordinate frames, the dynamic model of the surface vessels can be described as [32-33]:

$$
\begin{aligned}
& \dot{\eta}=J(\eta) v \\
& M \dot{v}+C(v) v+D(v) v+G(\eta)+F(\eta, v, t)=\tau
\end{aligned}
$$

where $\eta=\left[\begin{array}{lll}x & y & \psi\end{array}\right]^{T} \in \mathfrak{R}^{3}$ is a vector that represents the Earth-frame position $(x, y)$ and heading $\psi \in[0,2 \pi]$, respectively, the vector $v=\left[\begin{array}{lll}v_{x} & v_{y} & v_{\psi}\end{array}\right]^{T} \in \mathfrak{R}^{3}$ denotes the velocity of the surface vessels in surge, sway and yaw direction in the body fixed frame, respectively. $M \in \mathfrak{R}^{3 \times 3}$ is a symmetric positive definite inertia matrix, $C(v) \in \mathfrak{R}^{3 \times 3}$ is the Centripetal and Coriolis matrix torques, which describes the rate of the required force to obtain the same acceleration in the rotating frame. $D(v) \in \mathfrak{R}^{3 \times 3}$ is the damping matrix, which represents forces from skin friction, wave-drift damping and damping due to vortex shedding. $G(\eta) \in \mathfrak{R}^{3}$ is a vector that represents the gravitational forces. $\tau \in \mathfrak{R}^{3}$ is the vector control input, which consists of the surge force, the sway force and the yaw moment. The vector $F(\eta, v, t) \in \mathfrak{R}^{3}$ denotes the lumped model uncertainties in the system, and $J(\eta)$ is the rotation matrix defined as $J(\eta)=\left[\begin{array}{ccc}\cos \psi & -\sin \psi & 0 \\ \sin \psi & \cos \psi & 0 \\ 0 & 0 & 1\end{array}\right]$.

Let $x_{1}=\eta, x_{2}=\dot{\eta}$, then, from (1) and (2), the dynamics model of the surface vessel can be described as

$$
\begin{aligned}
\dot{x}_{1} & =x_{2} \\
\dot{x}_{2} & =\dot{J}(\eta) v+J(\eta) M^{-1} \tau+J(\eta) M^{-1}(-C(v) v-D(v) v-G(\eta)-F(\eta, v, t)) \\
& =\Xi \tau+\Xi(-C(v) v-D(v) v-G(\eta))+\dot{J}(\eta) v+\Xi(F(\eta, v, t)) \\
& =\Xi u+\Gamma(\eta, v)+\Psi(\eta, v, t)
\end{aligned}
$$

where $u=\tau, \Xi=J(\eta) M^{-1}, \Gamma(\eta, v)=\Xi(-C(v) v-D(v) v-G(\eta))+\dot{J}(\eta) v$ denotes the nominal component and $\Psi(\eta, v, t)=\Xi(-F(\eta, v, t))$ denotes the lumped uncertainty in the new defined dynamics model (3).

The expression in (3) can be rewritten in shorter form:

$\dot{x}_{1}=x_{2}$

$\dot{x}_{2}=\Xi u+\Gamma(\eta, v)+\Psi(\eta, v, t)$

Assumption 1: the lumped model uncertainty is bounded: 
where $\chi$ is an unknown constant. This assumption means that the unknown components in the dynamics system such as structured uncertainties, friction, external disturbance, etc., are bounded, and therefore the system states are bounded. This assumption is usually satisfied in the practical engineering applications.

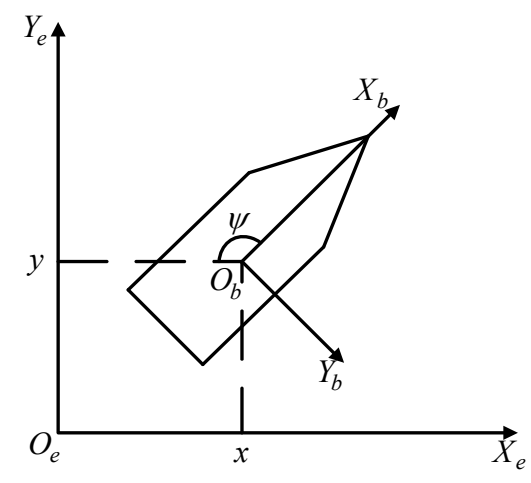

Figure 1. Definition of reference coordinate frames of the vessel motion.

Remark 1: Generally, the lumped model uncertainties, i.e., $F(\eta, v, t)$, of the dynamics model of the surface vessels can be expressed as: $F(\eta, v, t)=f_{1}(\eta, v, t)+f_{2}(\eta, v, t)-\tau_{d}(t)$, where $f_{1}(\eta, v, t)+f_{2}(\eta, v, t)$ is used to represent the unmodeled dynamics of the system, in which $f_{1}(\eta, v, t)$ denotes the structured uncertainty, i.e., the difference between the calculated nominal parameters and the real parameters of the matrices $C(v), D(v)$, $G(\eta)$, the component $f_{2}(\eta, v, t)$ is used to represent the unstructured uncertainties like friction, etc., in the system, and $\tau_{d}(t)$ is used to express the effects of the external disturbance forces, i.e., wind, waves and currents forces. For the sake of design of controller, all the unknown components in the system are represented by the lumped uncertainties $F(\eta, v, t)$ only. Since the vessels work in the unstructured environment, particularly in harsh sea condition, where the effects of the external disturbances, i.e., wind, waves and currents, are very strong, the tracking performance of the system is significantly decreased. Hence, it is necessary to further develop effective controllers to enhance the tracking performance of the system.

\section{Design of Integral Sliding Mode Control for Tracking Control of Surface Vessels}

Let $e=x_{1}-x_{d}$ is the trajectory tracking error, where $x_{d}=\eta_{d}=\left[\begin{array}{lll}x_{d} & y_{d} & \psi_{d}\end{array}\right]^{T}$ denotes the desired trajectory. First, the following filter error is reconstructed as: 
$s=\dot{e}+\lambda e$

where $\lambda$ is a constant.

Differentiating (6) with respect to time and using (4), we have

$\dot{s}=\ddot{e}+\lambda \dot{e}=\Xi u+\Gamma(\eta, v)+\Psi(\eta, v, t)-\ddot{x}_{d}+\lambda \dot{e}$

Based on the system (7), the integral sliding surface is selected as [21, 23]:

$\sigma(t)=s(t)-s(0)-\int_{0}^{t}\left(\Xi u_{0}+\Gamma(\eta, v)-\ddot{x}_{d}+\lambda \dot{e}\right) d t$

where $s(0)$ defines the value of the sliding surface at the time $t=0$. The term of $-s(0)$ is added to get the exciting property that $\sigma(0)=0$. It means the reaching phase is eliminated. $u_{0}$ is the nominal controller, which is designed to stabilize the nominal system (the system without the lumped uncertainty, i.e., $\left.\dot{s}=\Xi u+\Gamma(\eta, v)-\ddot{x}_{d}+\lambda \dot{e}\right)$.

Differentiating the sliding surface (8) with respect to time, we have

$\dot{\sigma}=\left(\Xi u+\Gamma(\eta, v)+\Psi(\eta, v, t)-\ddot{x}_{d}+\lambda \dot{e}\right)-\left(\Xi u_{0}+\Gamma(\eta, v)-\ddot{x}_{d}+\lambda \dot{e}\right)$

The control input can be chosen as the following form:

$u=u_{0}+u_{s}$

where the switching term is selected as follows:

$u_{s}=-\Xi^{-1}(\chi+\vartheta) \operatorname{sign}(\sigma)$

where $\vartheta$ is a small positive constant and $\chi$ is selected as in the Assumption 1 .

Inserting the composite controller (10) and (11) into (9), we have

$\dot{\sigma}=-(\chi+\vartheta) \operatorname{sign}(\sigma)+\Psi(\eta, v, t)$

Define a Lyapunov function candidate $V=\frac{1}{2} \sigma^{T} \sigma$. Differentiating the Lyapunov function, we have

$$
\begin{aligned}
\dot{V} & =\sigma \dot{\sigma} \\
& =\sigma(-(\chi+\vartheta) \operatorname{sign}(\sigma)+\Psi(\eta, v, t)) \\
& =-(\chi+\vartheta)|\sigma|+\Psi(\eta, v, t) \sigma \\
& <0
\end{aligned}
$$

Therefore, based on Lyapunov criterion, we can verify that the stability of the sliding surface is guaranteed. 


\section{Design of Neural Integral Sliding Mode Control for Tracking Control of Surface Vessels}

In the design of the ISMC for surface vessels in section 3, the sliding gain was chosen based on the assumption that the bounded value of the lumped uncertainty could be known in advance. However, this assumption is difficult to be obtained in advance in real implementations. In addition, suppose that the bounded value can be obtained but big, the sliding gain must be chosen as a big value to guarantee the existing condition of the integral sliding mode control. Unfortunately, since the chattering is proportional to the magnitude of the sliding gain, this approach provides big chattering. In order to eliminate the Assumption 1 and chattering, this section presents the proposed method based on a hybrid NN and ISMC.

\subsection{Radial Basis Function Neural Network and Lemmas}

The radial basis function neural network $(\mathrm{RBFNN})$ is a three-layer feedforward neural network, consisting of one input layer, one hidden layer, and one output layer. The output of the RBFNN can be defined as follows [3233]:

$F(Z)=W^{* T} \Theta(Z)+\delta$

where $Z$ and $F(Z)$ are the input and output of the RBFNN, respectively. $W^{*} \in \mathfrak{R}^{n \times l}$ is the weight matrix of the hidden nodes. $\Theta(Z) \in \mathfrak{R}^{l}$ is the nonlinear function of the hidden nodes. $\delta \in \mathfrak{R}^{n}$ is an approximation error with bound. A Gaussian function is chosen for the nonlinear function:

$\Theta(Z)=\exp \left(\frac{-\left(Z-\mu_{i}\right)^{T}\left(Z-\mu_{i}\right)}{\varphi_{i}^{2}}\right), i=1,2, \ldots, l$

where $\mu$ and $\varphi$ are the center and width of the Gaussian function, respectively.

Lemma 1[32-33]: For any given real continuous function $F(Z)$ on a compact set $U_{Z} \in \mathfrak{R}^{n}$ and an arbitrary $\delta>0$, there exists an integer $W^{* T} \Theta(Z)$, such that

$$
\sup _{Z \in U_{Z}}\left|W^{* T} \Theta(Z)-F(Z)\right| \leq \delta
$$

Lemma 2 [30]: Consider a nonlinear system $\dot{x}=f(x)$. Suppose that there exists a smooth positive definite function $V(x)>0$ and some scalars $a>0$ and $b>0$ such that

$$
\dot{V}(x) \leq-a V(x)+b
$$

Then, the solution $x(t)$ is uniformly bounded. 


\subsection{Design of hybrid integral sliding mode control and neural network}

Based on the approximation capability of $\mathrm{NN}$, the lumped model uncertainty in (4) can be represented as:

$\Psi(\eta, v, t)=W^{* T} \Theta(Z)+\delta$

where $W^{*}, \Theta(Z), Z=[\eta, \nu]^{T}$ are the NN's weight vector, the radial basis function and the input vector of NN, respectively. $\delta$ is the approximation error.

Assumption 2: The NN's approximation error $\delta$ is bounded:

$\|\delta\| \leq \kappa$

where $\kappa$ is an unknown constant.

From (4) and (18), the dynamics model of the system can be rewritten as:

$\dot{x}_{1}=x_{2}$

$\dot{x}_{2}=\Xi u+\Gamma(\eta, v)+W^{*} T \Theta(Z)+\delta$

Differentiating (6) with respect to time and using (20), we have

$\dot{s}=\ddot{e}+\lambda \dot{e}=\Xi u+\Gamma(\eta, v)+W^{* T} \Theta(Z)+\delta-\ddot{x}_{d}+\lambda \dot{e}$

In the system (21), the new nominal system is defined as $\Omega=\Xi u_{0}+\Gamma(\eta, v)+W^{*} T \Theta(Z)-\ddot{x}_{d}+\lambda \dot{e}$, and the NN's approximation error, i.e., $\delta$, is an unknown component. Based on (21), the integral sliding mode surface is selected as:

$\sigma(t)=s(t)-s(0)-\int_{0}^{t}\left(\Xi u_{0}+\Gamma(\eta, v)+W^{* T} \Theta(Z)-\ddot{x}_{d}+\lambda \dot{e}\right) d t$

where $s(0)$ defines the value of the sliding surface at the time $t=0 . u_{0}$ is the nominal controller, which will be designed later, to stabilize the new nominal system, i.e., $\Omega$.

Differentiating the sliding surface (22) with respect to time, we have

$\dot{\sigma}=\left(\Xi u+\Gamma(\eta, v)+W^{*} T \Theta(Z)+\delta-\ddot{x}_{d}+\lambda \dot{e}\right)-\left(\Xi u_{0}+\Gamma(\eta, v)+W^{*} T \Theta(Z)-\ddot{x}_{d}+\lambda \dot{e}\right)$

The control input is now proposed as:

$u=u_{0}+u_{s}$

where $u_{0}$ is the nominal controller, which is used to stabilize the nominal system and will be designed in the next subsection. The switching term is selected as follows:

$u_{S}=-\Xi^{-1}(\kappa+\vartheta) \operatorname{sign}(\sigma)$ 
where $\vartheta$ is a small positive constant and $\kappa$ is selected as in Assumption 2.

Inserting the composite controller (24) and (25) into (23), we have

$\dot{\sigma}=-(\kappa+\vartheta) \operatorname{sign}(\sigma)+\delta$

Define a Lyapunov function candidate $V=\frac{1}{2} \sigma^{T} \sigma$. Differentiating the Lyapunov function, we have

$$
\begin{aligned}
\dot{V} & =\sigma \dot{\sigma} \\
& =\sigma(-(\kappa+\vartheta) \operatorname{sign}(\sigma)+\delta) \\
& =-(\kappa+\vartheta)|\sigma|+\delta \sigma \\
& <0
\end{aligned}
$$

Therefore, based on Lyapunov criterion, we can verify that the stability of the sliding surface is guaranteed.

Remark 2: The employed of the NN in this paper is different from the conventional approaches in [32-37]. In the approaches in [32-37], the $\mathrm{NN}$ is used as the feedforward compensation term. In contrast, in this paper, the adaptive $\mathrm{NN}$ is employed in both the design of the sliding surface and feedforward compensation term in the design of the nominal controller.

\subsection{Design of nominal controller based on backstepping control technique for the nominal system}

From (21), the nominal system can be rewritten as

$$
\begin{aligned}
& \dot{x}_{1}=x_{2} \\
& \dot{x}_{2}=\Xi u_{0}+\Gamma(\eta, v)+W^{*} T \Theta(Z)
\end{aligned}
$$

In order to guarantee the asymptotic stability of the nominal system, the following backstepping design procedure is developed for the system (28):

Step 1: The following is defined as the first sliding error:

$z_{1}=x_{1}(t)-x_{d}(t)$

Differentiating (29) with respect to time, we obtain

$\dot{z}_{1}=\alpha_{1}(t)-\dot{x}_{d}(t)$

where $\dot{x}_{1}=\alpha_{1}(t)$ can be considered as virtual control in the above equation. Next, the following stabilizing function is defined as:

$\alpha_{1}(t)=-k_{1} z_{1}(t)+\dot{x}_{d}(t)$

where $k_{1}$ is a positive constant.

Step 2: In this step, we define second sliding error as below

$z_{2}(t)=x_{2}(t)-\alpha_{1}(t)$ 
Then, the derivative of $z_{2}(t)$ with respect to time is computed as

$$
\begin{aligned}
\dot{z}_{2}(t) & =\dot{x}_{2}(t)-\dot{\alpha}_{1}(t) \\
& =\Xi u_{0}+\Gamma(\eta, v)+W^{* T} \Theta(Z)+k_{1} \dot{z}_{1}(t)-\ddot{x}_{d}(t)
\end{aligned}
$$

Remark 3: From (22) and (33), we can see that $\dot{z}_{2}(t)$ equals to $\Omega$, i.e., $\dot{z}_{2}(t)=\Omega$, when $k_{1}=\lambda$. Consequently, if the nominal controller, i.e., $u_{0}$, can stabilize $\dot{z}_{2}(t)$, then we can conclude that $u_{0}$ can guarantee the stability of the nominal system $\Omega$. Therefore, the backstepping control technique developed in this subsection can be used for the nominal controller of (22).

Step 3: In this step, a Lyapunov function is defined as below:

$$
V\left(z_{1}, z_{2}, \tilde{\theta}\right)=\frac{1}{2} z_{1}^{2}+\frac{1}{2} z_{2}^{2}+\frac{1}{2} \tilde{\theta}^{2}
$$

where $\tilde{\theta}=\theta-\hat{\theta}$ is the estimation error with $\theta=\max _{l \in L}\left\|W_{l}^{*}\right\|(l$ is the number of nodes of NN) and $\hat{\theta}$ is the estimation of $\theta$.

Differentiating the Lyapunov function with respect to time, we have

$$
\begin{aligned}
\dot{V}\left(z_{1}, z_{2}, \tilde{\theta}\right) & =z_{1} \dot{z}_{1}+z_{2} \dot{z}_{2}-\tilde{\theta} \dot{\hat{\theta}} \\
& =z_{1}\left(z_{2}(t)+\alpha_{1}(t)-\dot{x}_{d}(t)\right)+z_{2}\left(\Xi u_{0}+\Gamma(\eta, v)+W^{*} T \Theta(Z)+k_{1} \dot{z}_{1}(t)-\ddot{x}_{d}(t)\right)-\tilde{\theta} \dot{\hat{\theta}}
\end{aligned}
$$

Applying the Young's inequality, one has [34, 35]

$z_{2} W^{* T} \Theta(Z) \leq \frac{1}{2}+\frac{1}{2} z_{2}^{2} \theta \Theta(Z) \Theta(Z)$

Then, $\dot{V}$ becomes

$$
\begin{aligned}
\dot{V}\left(z_{1}, z_{2}, \tilde{\theta}\right) & \leq z_{1} \dot{z}_{1}+z_{2} \dot{z}_{2}-\tilde{\theta} \dot{\hat{\theta}} \\
& \leq z_{1}\left(z_{2}(t)+\alpha_{1}(t)-\dot{x}_{d}(t)\right)+z_{2}\left(\Lambda u_{0}+\Gamma(\eta, v)+\frac{1}{2} z_{2} \theta \Theta^{T}(Z) \Theta(Z)+k_{1} \dot{z}_{1}(t)-\ddot{x}_{d}(t)\right)+\frac{1}{2}-\tilde{\theta} \dot{\hat{\theta}} \\
& \leq z_{1}\left(z_{2}(t)+\alpha_{1}(t)-\dot{x}_{d}(t)\right)+z_{2}\left(\Lambda u_{0}+\Gamma(\eta, v)+\frac{1}{2} z_{2} \hat{\theta} \Theta \Theta^{T}(Z) \Theta(Z)+k_{1} \dot{z}_{1}(t)-\ddot{x}_{d}(t)\right) \\
& +\frac{1}{2}+\tilde{\theta}\left(\frac{1}{2} z_{2}^{2} \Theta^{T}(Z) \Theta(Z)-\dot{\hat{\theta}}\right)
\end{aligned}
$$

Then, the following control input is designed for the nominal system

$u_{0}=\Xi^{-1}\left(-\Gamma(\eta, v)-\frac{1}{2} z_{2} \hat{\theta}^{T}(Z) \Theta(Z)-k_{1} \dot{z}_{1}(t)+\ddot{x}_{d}(t)-z_{1}-k_{2} z_{2}\right)$

and the adaptive gain is selected as

$\dot{\hat{\theta}}=\frac{1}{2} z_{2}^{2} \Theta^{T}(Z) \Theta(Z)-2 \gamma \hat{\theta}$

where $\gamma$ is a design parameter. 
Inserting (38) and (39) into (37), one yields

$$
\begin{aligned}
\dot{V}\left(z_{1}, z_{2}, \tilde{\theta}\right) & \leq z_{1} \dot{z}_{1}+z_{2} \dot{z}_{2}-\tilde{\theta} \dot{\hat{\theta}} \\
& \leq-k_{1} z_{1}^{2}-k_{2} z_{2}^{2}+\frac{1}{2}+2 \gamma \tilde{\theta} \hat{\theta}
\end{aligned}
$$

Using the Young's inequality, one obtains $[34,35]$

$2 \gamma \tilde{\theta} \hat{\theta} \leq-\gamma \tilde{\theta}^{2}+\gamma \theta^{2}$

Inserting (41) into (40), it yields

$$
\begin{aligned}
\dot{V}\left(z_{1}, z_{2}, \tilde{\theta}\right) & \leq z_{1} \dot{z}_{1}+z_{2} \dot{z}_{2}-\tilde{\theta} \dot{\hat{\theta}} \\
& \leq-k_{1} z_{1}^{2}-k_{2} z_{2}^{2}-\gamma \tilde{\theta}^{2}+\frac{1}{2}+\gamma \theta^{2} \\
& \leq-a V+b
\end{aligned}
$$

where $a=\min \left(2 \lambda_{\min }\left(k_{1}\right), 2 \lambda_{\min }\left(k_{2}\right), 2 \lambda_{\min }(\gamma)\right), b=\frac{1}{2}+\gamma \theta^{2}>0 . \lambda_{\min }(\cdot)$ represents the minimum eigenvalue of a matrix. Iterating the inequality (42) from 0 to $t$, we obtain

$0 \leq V(t) \leq \frac{b}{a}+\left(V(0)-\frac{b}{a}\right) e^{-a t}$

It implies that $V$ is uniformly ultimately bounded. From (42) and Lemma 2, we conclude that $z_{1}, z_{2}, \tilde{\theta}$ are bounded for bounded initial conditions.

Remark 4: It is worth mentioning that the developed integral sliding mode control in this paper is far different from the so-called integral sliding mode control used in $[40,41]$. Consequently, the developed hybrid backstepping control, ISMC and adaptive NN in this paper is far different from the one developed in [43, 44]. In the approaches in $[40,41]$ and $[43,44]$, only integral of the tracking error is used in the design of the sliding surface. The goal of the approaches is to enhance the robustness of the SMC and reduce the steady-state error of the system by adding the integration of the error term into the system, while other properties are similar to the conventional SMC. In contrast, the ISMC developed in this paper used the integration of the nominal system, and therefore, the system can get the three exciting benefits as discussed in the introduction.

Remark 5: In the conventional adaptive NN, $l$ adaptive rules are needed to adapt $l$ NN's weights, which will increase the computational burden of the system. To reduce the computational load of the system, young's inequality is employed in this paper. According to the rule defined in (37), only one adaptive rule is used for the system. In this sense, the sliding surface (22) can be replaced by the following sliding surface:

$\sigma(t)=s(t)-s(0)-\int_{0}^{t}\left(\Xi u_{0}+\Gamma(\eta, v)+\hat{\theta} \Theta(Z)-\ddot{x}_{d}+K \dot{e}\right) d t$ 


\subsection{Design of adaptive law for the integral sliding mode control}

In the developed ISMC presented in section 4.1, the sliding gain is chosen based on the Assumption 2. It means that the NN's approximation error must be known in advance. However, this parameter is difficult to be predicted in advance since this value depends on many factors such as the number of nodes of $\mathrm{NN}$, the variations of the lumped model uncertainty, and so on. Therefore, to remove the assumption in the design, an adaptive law is designed in this subsection to estimate the NN's approximation error $\kappa$.

The control input is now proposed as

$u=u_{0}+u_{a s}$

where the nominal controller is designed as in (38) and (39), and the switching term is modified as:

$u_{\text {as }}=-\Xi^{-1}(\hat{\kappa}+\vartheta) \operatorname{sign}(\sigma)$

where $\hat{\kappa}$ is the estimation of the NN's approximation error $\kappa$. This estimation is adapted by using the following law:

$\dot{\hat{\kappa}}=\frac{1}{\rho}|\sigma|$

where $\rho$ denotes the adaptation gain.

Consider a Lyapunov function candidate:

$V=\frac{1}{2} \sigma^{T} \sigma+\frac{1}{2} \rho \tilde{\kappa}^{T} \tilde{\kappa}$

where $\tilde{\kappa}=\hat{\kappa}-\kappa$ is the adaptation error.

Differentiating the above Lyapunov function and using (23), (45)-(47), we obtain

$$
\begin{aligned}
\dot{V} & =\sigma \dot{\sigma}+\rho \tilde{\kappa} \dot{\tilde{\kappa}} \\
& =\sigma\left(\Xi u_{a s}+\delta\right)+\rho(\hat{\kappa}-\kappa) \dot{\hat{\kappa}} \\
& =\sigma(-(\hat{\kappa}+\vartheta) \operatorname{sign}(\sigma)+\delta)+(\hat{\kappa}-\kappa)|\sigma| \\
& \leq-\vartheta|\sigma|+\delta \sigma-\kappa|\sigma| \\
& \leq-\vartheta|\sigma|
\end{aligned}
$$

Because $\vartheta>0, \dot{V}$ becomes negative semi-definite, i.e., $\dot{V} \leq-\vartheta|\sigma|$. Using Lyapunov criteria, the convergence of $\sigma$ and $\tilde{\kappa}$ to zero are guaranteed.

The block diagram of the developed controller is shown in Fig. 2.

Remark 6: In the switching term of the hybrid ISMC and NN described in (25), the sliding gain $\kappa$ is chosen based on the Assumption 2, whilst in the proposed ANISMC (46), the switching gain is adapted according to the law (46). Therefore, the advantage of the proposed ANISMC is that it can eliminate the Assumption 2 in the 
design and automatically update the switching gain.

Remark 7: From (47) $\left(\dot{\hat{\kappa}}=\frac{1}{\rho}|\sigma|\right)$, the adaptation gain $\hat{\kappa}$ keeps increasing until the sliding surface $\sigma$ equals to zero; this symptom is known as 'parameter drift problem'. Unfortunately, it is very difficult to achieve $\sigma=0$ due to the effects of noise and other imperfect parameters, and thus, the drift problem is always occurred. To prevent the parameter drift problem, the following dead-zone method is used:

$\dot{\hat{\kappa}}=\left\{\begin{array}{cc}0 & \text { if }|\sigma| \leq \varepsilon \\ \frac{1}{\rho}|\sigma| & \text { if }|\sigma|>\varepsilon\end{array}\right.$

where $\varepsilon$ is a (small) positive constant, which is selected based on experiment. The experiment can be done offline as follows. First, select the big value of the adaptation gain $\hat{\kappa}(\|\delta\| \leq \hat{\kappa})$ to guarantee the existing condition of the sliding mode and observer the sliding error, i.e., $\sigma$, when the system is converged. Then, calculate the bounded value $\sigma_{b}$ of the sliding error $\sigma$. The parameter $\varepsilon$ should be chosen to be bigger than the value of $\sigma_{b}$ to avoid the parameter drift problem.

The stability of the system under the adaptive law (50) can be explained as follows. First, when $|\sigma|>\varepsilon$, the stability of the system is guaranteed as in (49). In contrast, when $|\sigma| \leq \varepsilon$, then $\dot{\hat{\kappa}}=0$. It means that the adaptive gain $\hat{\kappa}$ converged to the true value $\kappa$, i.e., $\hat{\kappa} \equiv \kappa>|\delta|$. Consequently, the Lyapunov function in (49) can be rewritten as

$$
\begin{aligned}
\dot{V} & =\sigma \dot{\sigma} \\
& =\sigma\left(\Xi u_{\text {as }}+\delta\right) \\
& =\sigma(-(\hat{\kappa}+\vartheta) \operatorname{sign}(\sigma)+\delta) \\
& \leq-\vartheta|\sigma|+\delta \sigma-\hat{\kappa}|\sigma| \equiv-\vartheta|\sigma|+\delta \sigma-\kappa|\sigma| \\
& \leq-\vartheta|\sigma|
\end{aligned}
$$

Therefore, we can conclude that the stability of the system is always guaranteed for both $|\sigma| \leq \varepsilon$ and $|\sigma|>\varepsilon$.

Remark 8: It can be seen that the adaptive law in (50) remains unchanged or increased only (no decrease).

Therefore, to reduce the 'increase' of the adaptation gain due to the effects of disturbance, the suitable value of 
$\varepsilon$ needs to be selected, as discussed in Remark 7. Solving this issue is actually a motivated research topic in the development of adaptive control. This issue will be studied and addressed further in our future work. However, in the literature, some methods have been initially proposed to solve this problem. The interested readers are recommended to refer to $[45,46]$ for understanding the issue and solving the issue.

Remark 9: To further reduce the chattering, the sign function can be replaced by a sigmoid function, and thus the controller in (46) can be modified by [21]:

$$
u_{a s}=-\Xi^{-1}(\hat{\kappa}+\vartheta) \frac{\sigma}{|\sigma|+\xi}
$$

where $\xi$ is a small positive constant. It can be obtained that

$$
\lim _{\xi \rightarrow 0} \frac{\sigma}{|\sigma|+\xi}=\frac{\sigma}{|\sigma|}=\operatorname{sign}(\sigma)
$$

for $\sigma \neq 0$. Therefore, $\operatorname{sign}(\sigma) \approx \frac{\sigma}{|\sigma|+\xi}$ when $\xi$ is small. It verifies that the stability of the system is still guaranteed under the law (52) based on the result of the derivative of the Lyapunov function in (49). The selection of $\xi$ decides the trade-off between maintaining ideal performance and ensuring smooth control effort.

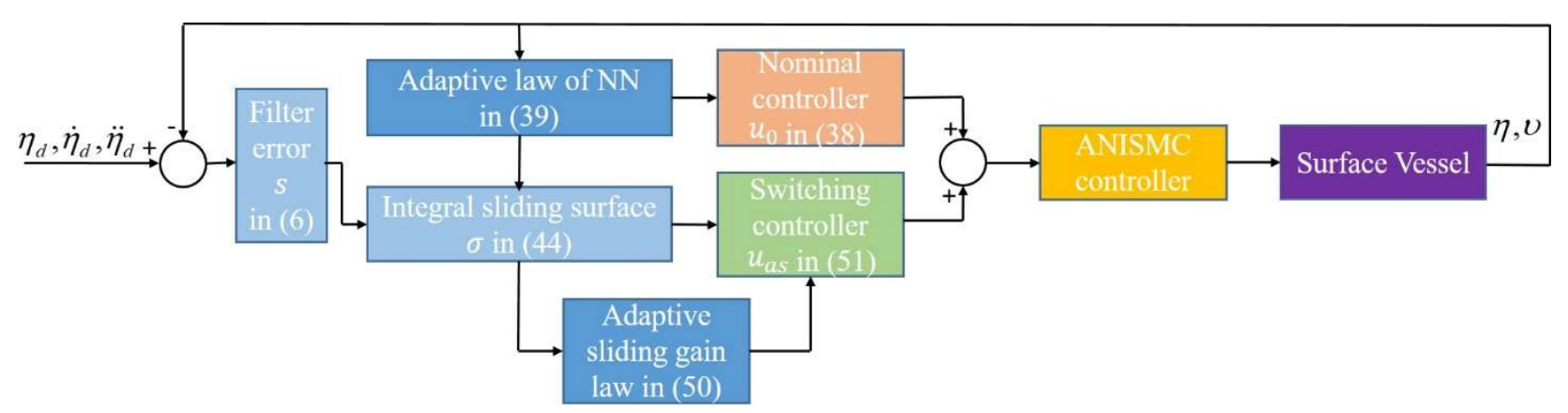

Figure 2. Overall structure of the proposed controller, i.e., ANISMC controller.

\section{Results and Discussions}

In order to verify the performance of the proposed method, we implement it in the simulated surface vessel based on Matlab/Simulink environment. The dynamic model used in this simulation can be described as in (1) and (2), where the parameters are selected as below [20]: 
$M=\left[\begin{array}{ccc}25.8000 & 0 & 0 \\ 0 & 24.6612 & 1.0948 \\ 0 & 1.0948 & 2.7600\end{array}\right]$

$C(v)=\left[\begin{array}{ccc}0 & 0 & -24.6612 v_{y} \\ 0 & 0 & 25.8 v_{x} \\ 24.6612 v_{y}+1.0948 v_{\psi} & -25.8 v_{x} & 0\end{array}\right]$

$G(\eta)=\left[\begin{array}{lll}0 & 0 & 0\end{array}\right]^{T}$

$D(v)=\left[\begin{array}{lll}D_{1} & D_{2} & D_{3}\end{array}\right]$

where:

$D_{1}(v)=\left[\begin{array}{c}0.7225+1.3274\left|v_{x}\right|+5.8664 v_{x}^{2} \\ 0 \\ 0\end{array}\right], D_{2}(v)=\left[\begin{array}{c}0 \\ 0.8612+36.2823\left|v_{y}\right|+8.05\left|v_{\psi}\right| \\ -0.1025-5.0437\left|v_{y}\right|-0.13\left|v_{\psi}\right|\end{array}\right]$,

$D_{3}(v)=\left[\begin{array}{c}0 \\ 0.845\left|v_{y}\right|+3.45\left|v_{\psi}\right|-0.1079 \\ 0.845\left|v_{y}\right|+3.45\left|v_{\psi}\right|-0.1079\end{array}\right]$

The surface vessel is commanded to track the following trajectory:

$\eta_{d}=\left[\begin{array}{l}x_{d} \\ y_{d} \\ \psi_{d}\end{array}\right]=\left[\begin{array}{c}4 \sin (0.02 t) \\ 2.5(1-\cos (0.02 t)) \\ 0.02 t\end{array}\right]$

The starting position and velocity of the vessels are selected as $\eta(0)=\left[\begin{array}{lll}0.5(\mathrm{~m}) & 0.5(\mathrm{~m}) & \pi / 9(\mathrm{rad})\end{array}\right]^{T}$ and $v(0)=\left[\begin{array}{lll}0(\mathrm{~m} / \mathrm{s}) & 0(\mathrm{~m} / \mathrm{s}) & 0(\mathrm{rad} / \mathrm{s})\end{array}\right]^{T}$.

In order to demonstrate the effectiveness of the proposed method, we compare it with three representative recent advanced control methods for tracking control of surface vessels, such as sliding mode control (SMC) [21], adaptive $\mathrm{NN}[34,35]$ (we applied the adaptive $\mathrm{NN}$ developed in $[34,35]$ for tracking control of surface vessel), which was used to design the nominal controller in section 4.2, and the adaptive backstepping sliding mode control (ABSMC), which has been proposed recently in [20]. In addition, we also compare the proposed method with the popular hybrid adaptive NN and SMC (ANSMC) [43]. The goal of the comparison with the 
SMC, the ABSMC and the ANSMC is to show the effectiveness of the ISMC, while the goal of the comparison with the adaptive $\mathrm{NN}$ is to show the high robustness of the proposed controller compared to the conventional NN. The parameters of the proposed adaptive neural integral sliding mode control (ANISMC) are selected as: $\lambda=[5,5,5]^{T}, k_{1}=[5,5,5]^{T}, k_{2}=[10,10,10]^{T}$, the center of NN $\mu$ is evenly spaced on $\mu=[-5,5]$ and the width is $\varphi=2$. The initial value of the adaptive law in (39) is $\hat{\theta}(0)=0$. The parameters for the adaptive law in (50) are selected as $\rho=0.2, \varepsilon=0.01$ and the initial adaptation value is $\hat{\kappa}(0)=3$, the parameter in (51) is chosen as $\xi=0.1$. The constant $\vartheta=0.1$. For fairer in comparison, the adaptive law (50) and the chattering reduction technique in (51) are also applied for the SMC.

In order to show the properties of the controllers, we consider the system in two operational conditions. First, a small uncertainty and disturbance is employed. Then, a big uncertainty and disturbance is considered.

First, the system is assumed to work in calm sea condition. For this condition, the following small uncertainty component is assumed:

$F_{1}(\eta, v, t)=\left[\begin{array}{c}-5 v_{x}^{2}-7 \sin \left(v_{x} v_{y}\right) \\ 6.1 v_{x}^{2}+7.5 \sin \left(v_{y}\right) \\ -3.2 v_{\psi}^{3}\end{array}\right]$

The uncertainty $F_{1}$ is a function of the velocities $v_{x}, v_{y}$ and $v_{\psi}$ only. Hence, it is used to present the model uncertainty like friction of the motor during rotating in the practical applications. In this condition, the external disturbance is assumed to be small, and therefore its effects can be neglected.

Uses five controllers: the ASMC, the ABSMC, the Adaptive NN, the ANSMC and the proposed ANISMC, one by one as the control input into the simulated surface vessel. The simulation results are shown in Figs. 3-4. Particularly, Fig. 3 shows the tracking performance of the controllers in the $(x-y)$ space. From Fig. 3, we can see that all the five controllers provide very good tracking performance for the system. However, as the results shown in Fig. 3, the Adaptive NN and the ANISMC converge faster than that of the ASMC, the ANSMC and the ABSMC. One interested point which can be picked out from Fig. 3 is that the response of the ANISMC is quite similar to the Adaptive NN; this is because we used the Adaptive NN for designing the nominal controller of the ANISMC. The tracking errors of the controllers are further shown in Fig. 4. For the sake of comparison, the rootmean-square-error (RMSE) is also reported in Table I, in which the parameters are defined as follows: 
$\left\|E_{x y}\right\|=\frac{1}{N} \sum_{i=1}^{N} \sqrt{\left(\left\|e_{x}(k)\right\|^{2}+\left\|e_{y}(k)\right\|^{2}\right)}$

$\left\|E_{\psi}\right\|=\frac{1}{N} \sum_{i=1}^{N} \sqrt{\left(\left\|e_{\psi}(k)\right\|^{2}\right)}$

where $N$ is the number of simulation steps, $e_{x}(k), e_{y}(k)$ and $e_{\psi}(k)$ are the tracking error in the $x$-axis, $y$-axis and $\psi$-axis, respectively.

From Fig. 4 and Table I, we can see that the ABSMC provides better performance than the SMC and the ANSMC. For example, the ABSMC provides 0.0013 and $4.6585 \times 10^{-4}$ for $(x y)$-axis and $\psi$-axis, respectively, while the corresponding values for the SMC are 0.0031 and 0.0028 , respectively and for the ANSMC are 0.0082 and 0.0070 , respectively. The responses of the ANISMC and the Adaptive NN are quite similar and both are better than the ABSMC, the ANSMC and the SMC. Particularly, the tracking errors of the ANISMC in (xy)-axis and $\psi$-axis are $3.9397 \times 10^{-4}$ and $1.9863 \times 10^{-4}$, respectively, while the corresponding values for the Adaptive NN are $4.2926 \times 10^{-4}$ and $2.1745 \times 10^{-4}$, respectively. The response of the integral sliding mode surface is show in Fig. 5. The result in Fig. 5 shows that the sliding surface of the ISMC converges to zero very quick (almost from the starting point). Based on this result, we can conclude that the proposed ANISMC is the best among controllers in both faster convergence speed and lower tracking error for this working condition.

Next, the system is assumed to work in harsh sea condition, and therefore the following lumped model uncertainty is assumed:

$$
F_{2}(\eta, v, t)=\left[\begin{array}{c}
10.3+50 \sin (0.5 t)+50 \sin (0.1 t) \\
-20.9+2 \sin (0.5 t-\pi / 6)+50 \sin (0.3 t) \\
-30 \sin (0.9 t+p i / 3)-30 \sin (0.1 t)
\end{array}\right]
$$

In the practical applications, the assumed uncertainty $F_{2}$ is used to represent the strong effects of the external disturbance from environment, i.e., strong wind, waves and currents, etc. Since the effects of the environment are unknown and difficult to be predicted, we used an arbitrary function as expressed in (60) to represent it.

The tracking performance of the controllers are shown in Figs. 6 and 7 and the RMSEs of the controllers are also reported in Table II. Particularly, Fig. 6 shows the trajectory tracking performance of the controllers. From Fig. 6, we can see that the SMC, the ANSMC and the Adaptive NN provide worst tracking response and precision for this working condition of the system. Due to the effects of the assumed uncertainties, there are oscillatory in the tracking errors of the SMC, the ANSMC and the Adaptive NN. On the other hand, as shown in Fig. 6, the 
proposed ANISMC converges faster and provides better tracking performance than the ABSMC, the ANSMC, the SMC and the Adaptive NN. Fig. 7 and Table II show the tracking errors of the system in three $x$-axis, $y$-axis and $\psi$-axis. From Fig. 7 and Table II, for the system with high uncertainty and disturbance, i.e., $F_{2}$, the Adaptive NN provides bigger tracking error. The ABSMC provides lower tracking error than the SMC, the ANSMC and the Adaptive NN. It should be noted that this result is different from the result for the system with the uncertainty $F_{1}$, where the Adaptive NN provided better tracking performance than the ABSMC. This means that, for the uncertainty $F_{2}$, which contains big unmodeled disturbance, the NN does not provide good approximation. Particularly, in (xy)-axis and $\psi$-axis, the RMSE of the ABSMC are 0.002 and 0.007 , respectively, while for the SMC are 0.0242 and 0.0351 , respectively, for the ANSMC are 0.0261 and 0.0369 , respectively, and for the Adaptive NN are 0.0232 and 0.0308 , respectively. On the other hand, from Fig. 7 and Table II, the proposed ANISMC provides superior performance. For example, in terms of convergence speed, the proposed ANISMC converges much faster than the ABSMC, the ANSMC, the SMC and the Adaptive NN, as shown in Fig. 7. In terms of tracking error, as shown in Table II, the ANISMC provides much lower tracking error compared to the ABSMC, the SMC, the ANSMC and the Adaptive NN. The convergences of the adaptive gains of the NN and the ISMC of the ANISMC are shown in Figs. 8 and 9, respectively. From Figs. 8 and 9 we can see that the adaptation gains of the ANISMC converge very quick. The control efforts of the controllers for the system with the uncertainty $F_{2}$ are shown in Fig. 10. It can be seen from Fig. 10 that all the control methods provide smooth control efforts and reasonable. It can be also seen that the range of the control efforts are between [-150N, $150 \mathrm{~N}]$. Therefore, based on the presented and analyzed results, we can conclude that when considering the performance of the controllers in terms of tracking precision, convergence speed, control efforts, the proposed method, i.e., ANISMC, is the best among the compared controllers in this simulation study. 

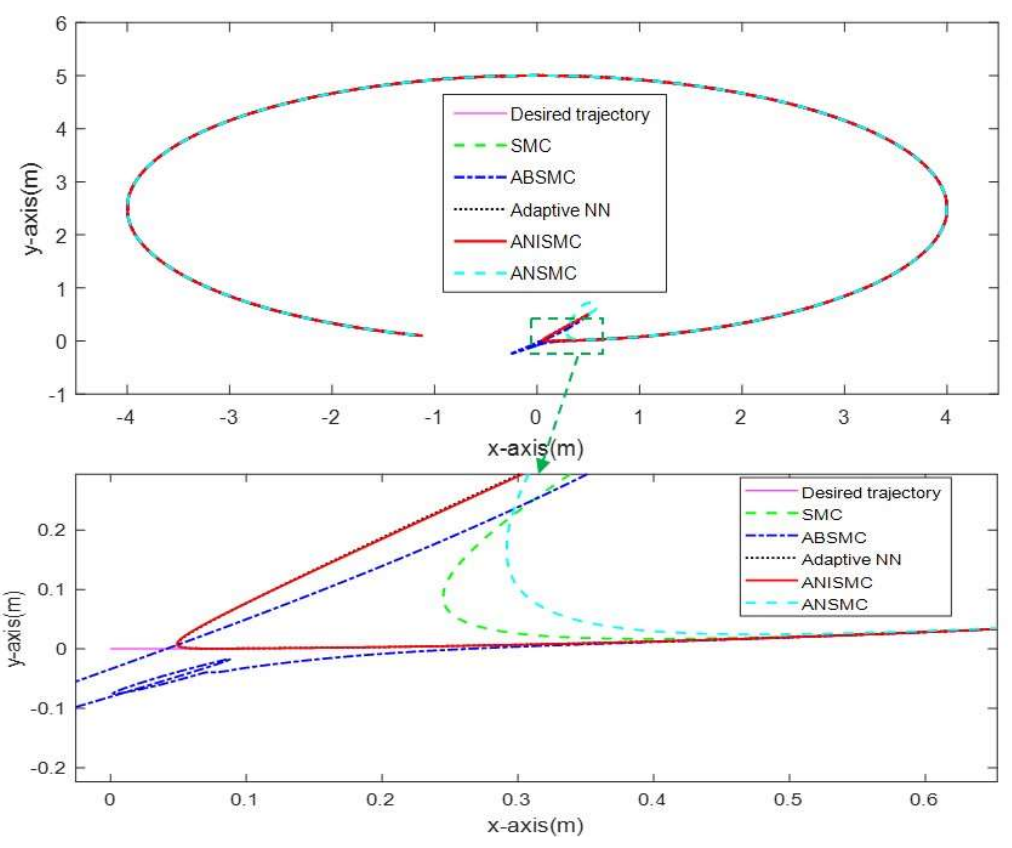

Figure 3. Desired trajectory and real tracking trajectory of the system with the presence of uncertainty $F_{1}$ under the SMC, the ABSMC, the Adaptive NN, the ANSMC and the ANISMC.

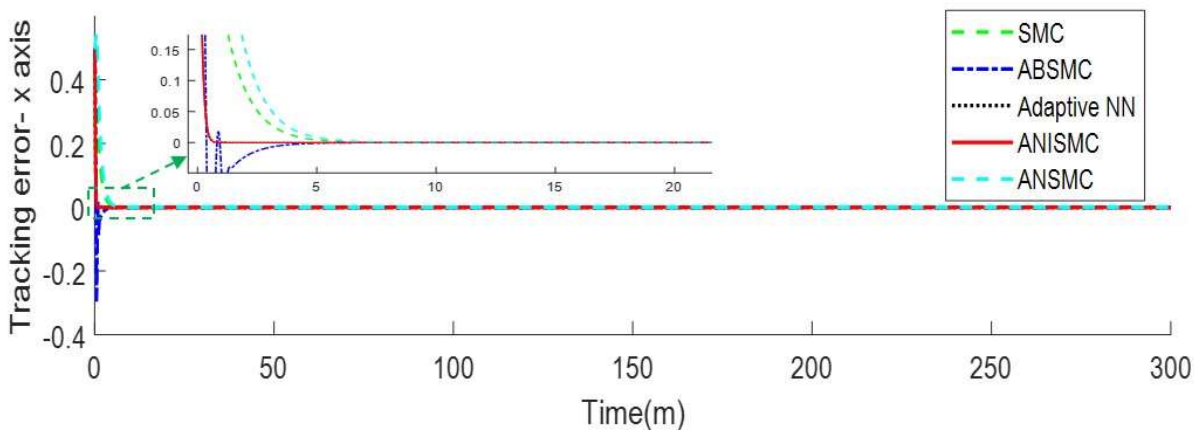

(a)

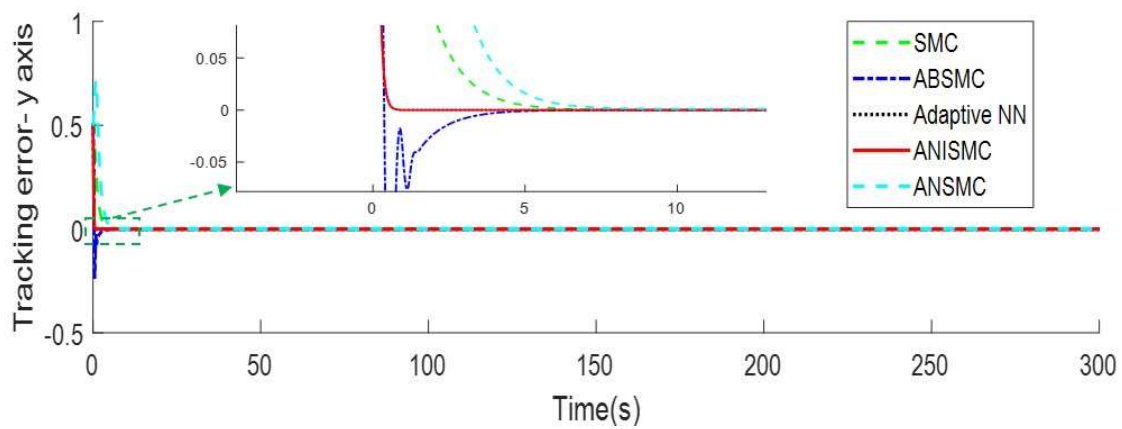

(b) 


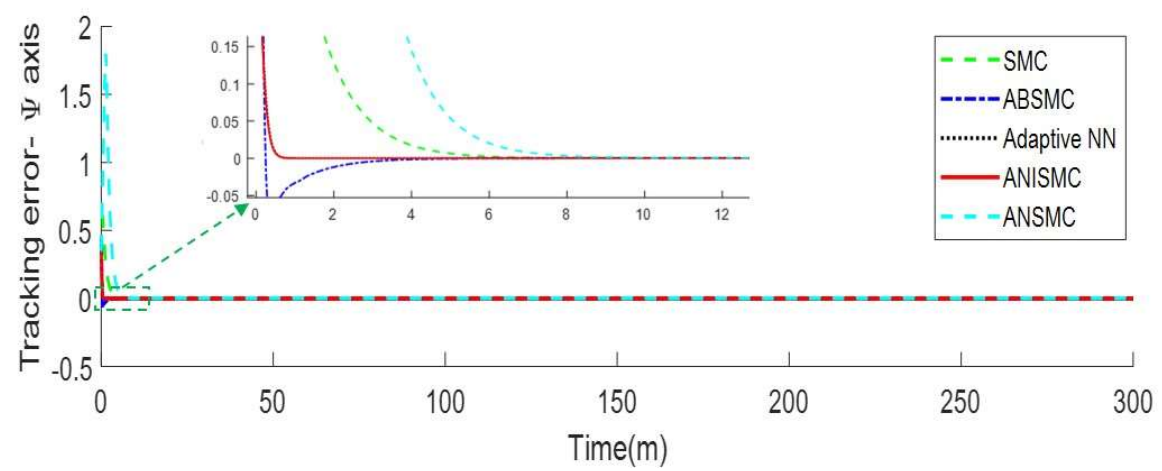

(c)

Figure 4. Comparison in tracking error in (a) $x$-axis, (b) $y$-axis and (c) $\psi$-axis among the SMC, the ABSMC, the Adaptive NN, the ANSMC and the ANISMC for the system with the presence of uncertainty $F_{1}$.

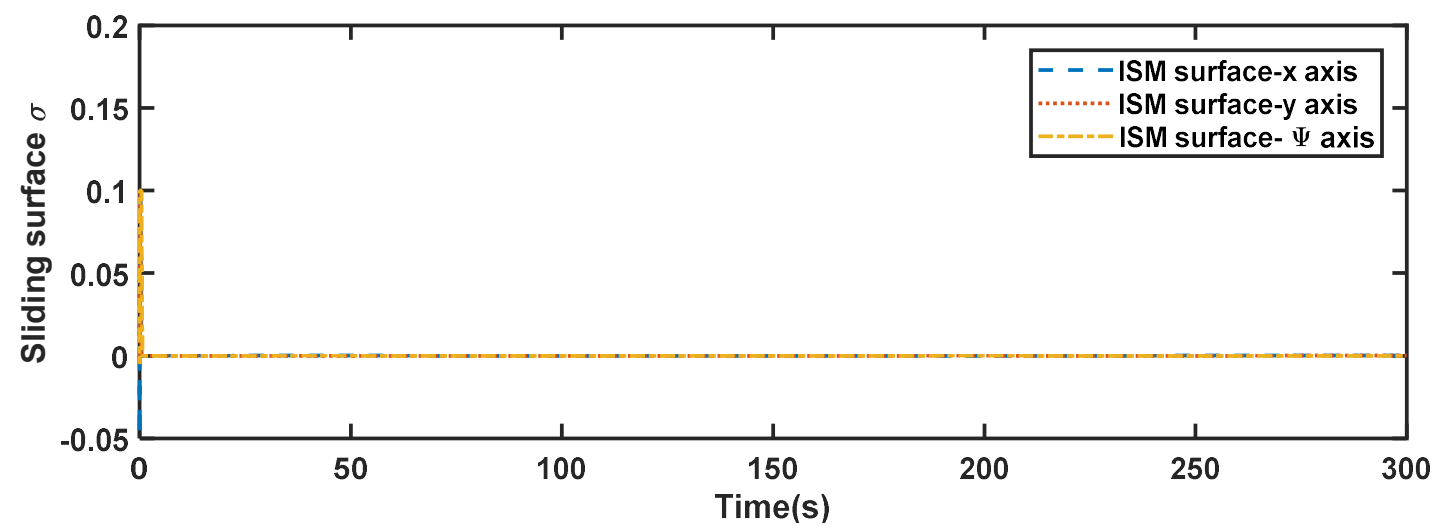

Figure 5. Sliding surface of the ISMC for the system with the presence of uncertainty $F_{1}$.
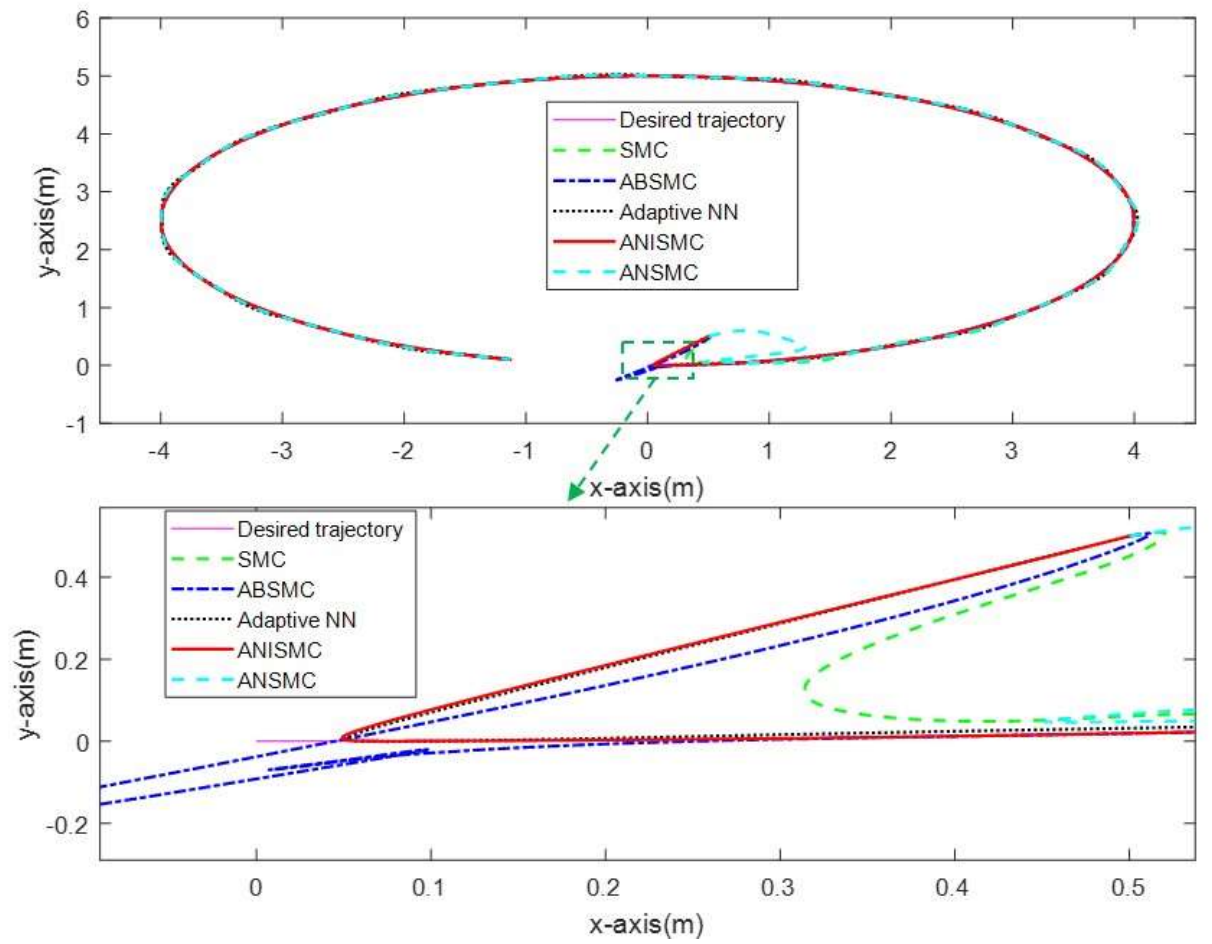
Figure 6. Desired trajectory and real tracking trajectory of the system with the presence of uncertainty $F_{2}$ under the SMC, the ABSMC, the Adaptive NN, the ANSMC and the ANISMC.

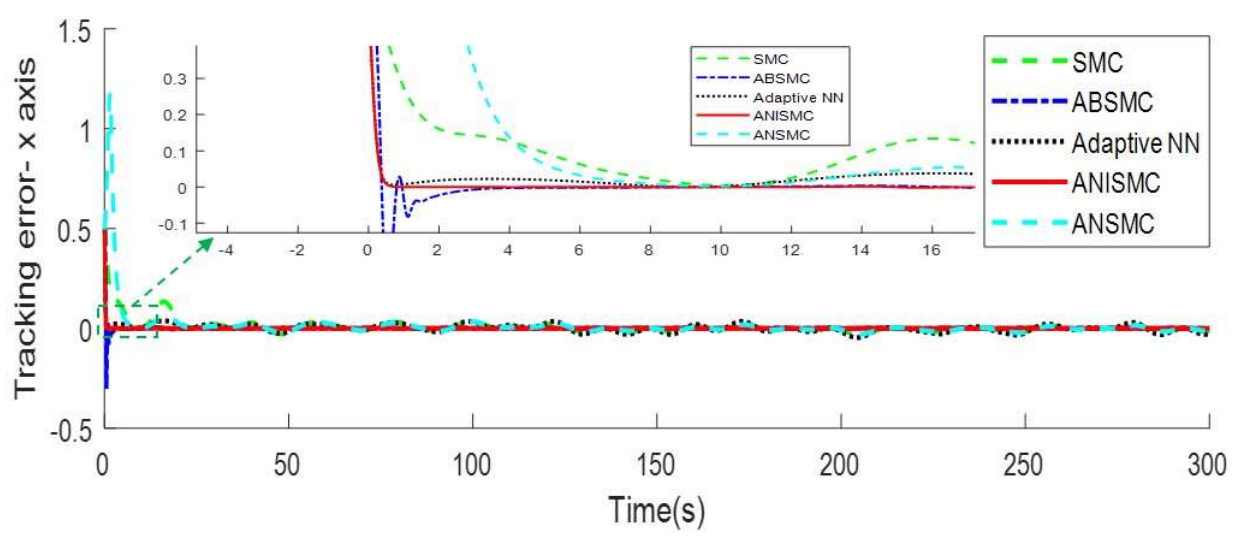

(a)

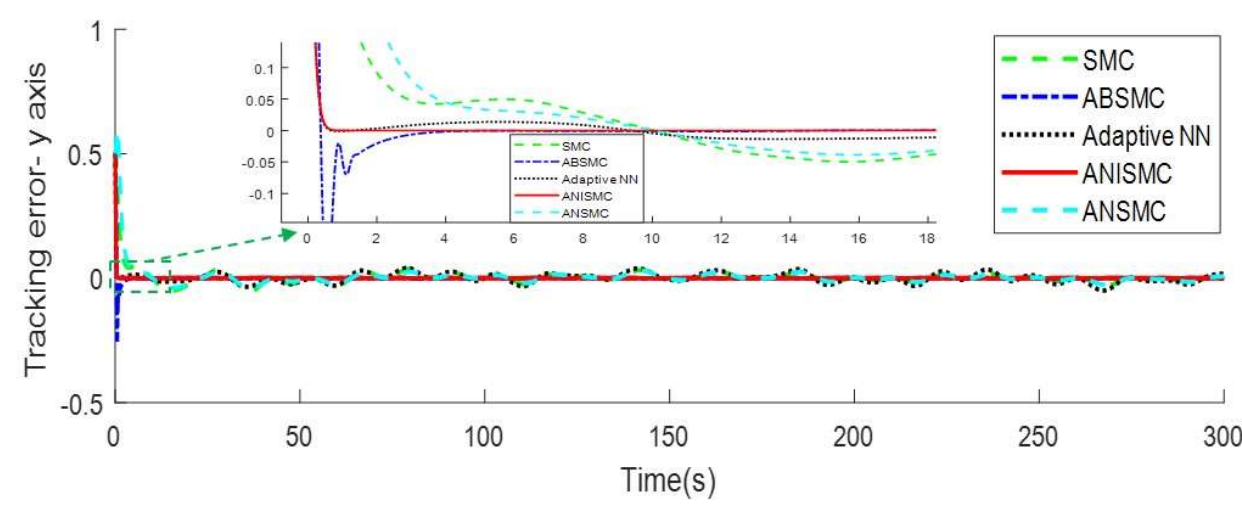

(b)

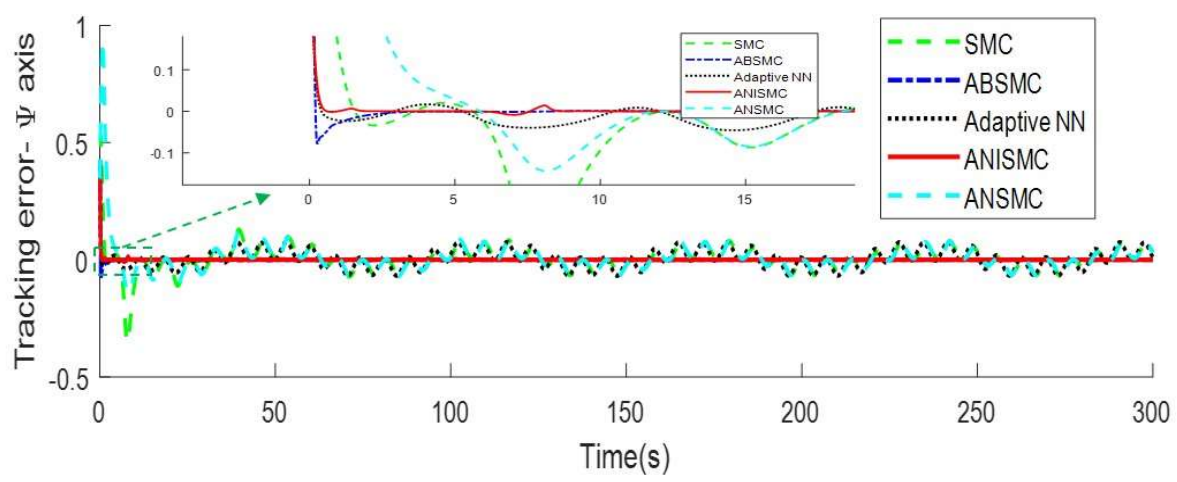

(c)

Figure 7. Comparison in tracking error in (a) $x$-axis, (b) $y$-axis and (c) $\psi$-axis among the SMC, the ABSMC, the Adaptive NN, the ANSMC and the ANISMC for the system with the presence of uncertainty $F_{2}$. 

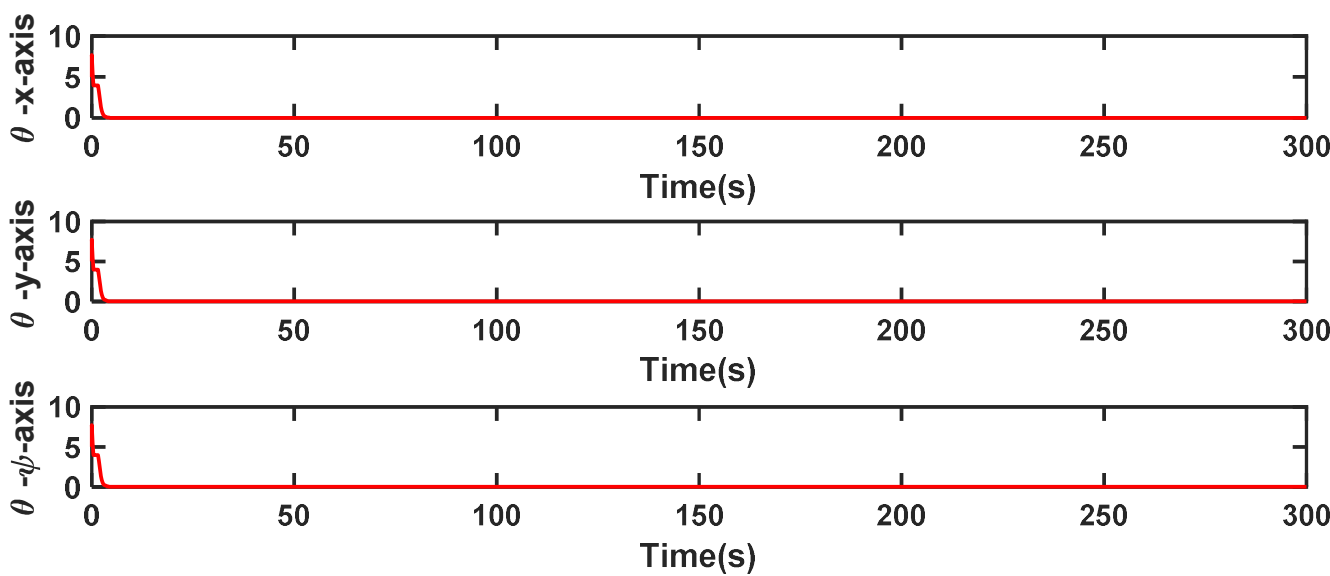

Figure 8. The responses of the adaptive gains of NN in (39) for the system with the presence of uncertainty $F_{2}$.

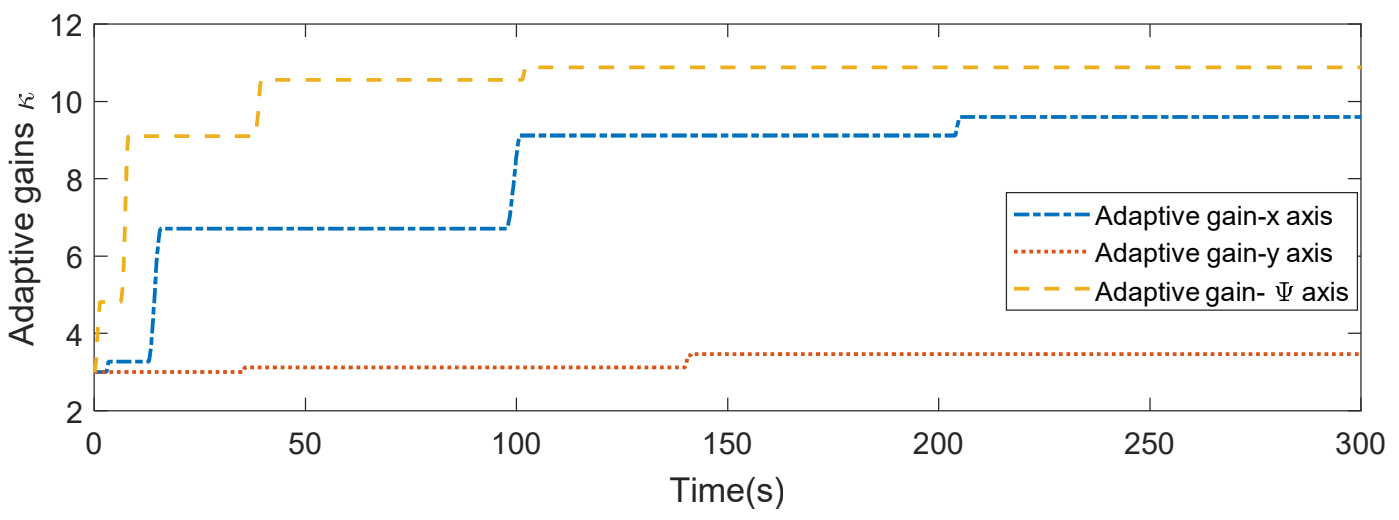

Figure 9. The responses of the adaptive sliding gains in (50) of the proposed ANISMC for the system with the presence of uncertainty $F_{2}$ when $\varepsilon=0.01$. 


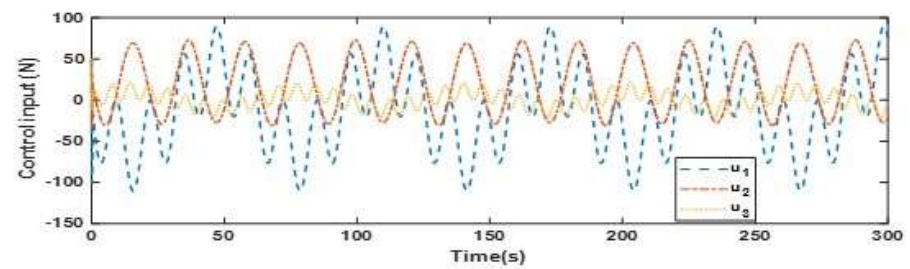

(a)

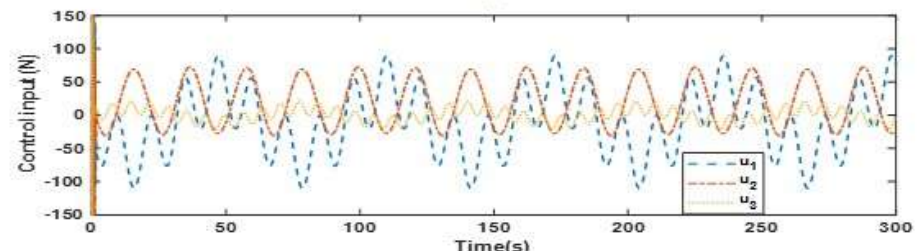

(b)

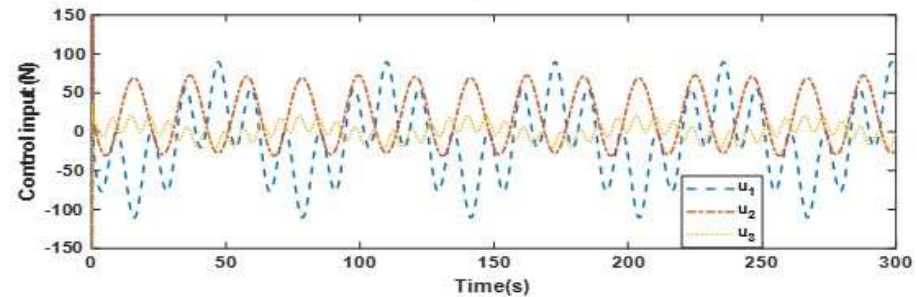

(c)

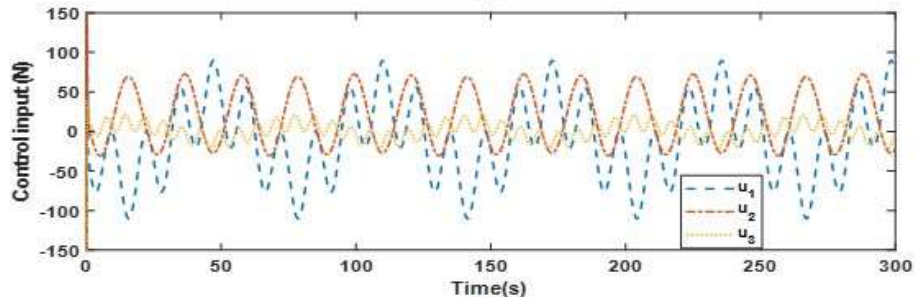

(d)

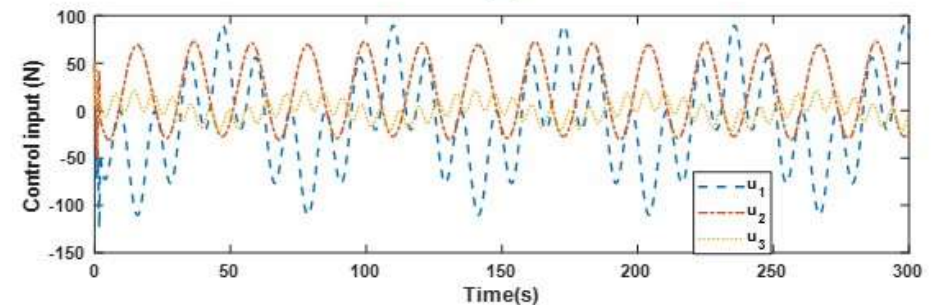

(e)

Figure 10. Control efforts of the controllers for the system with the presence of uncertainty $F_{2}$. (a) SMC, (b) ABSMC, (c) Adaptive NN, (d) ANISMC, and (e) ANSMC.

Table I: Comparison among the controllers in terms of root mean square error (RMSE) for the system with the uncertainty $F_{1}$

\begin{tabular}{l|cc}
\hline \multicolumn{1}{c|}{ RMSE } & $\left\|E_{x y}\right\|$ & $\left\|E_{\psi}\right\|$ \\
Controller & & \\
\hline SMC & 0.0031 & 0.0028 \\
ABSMC & 0.0013 & $4.6585 \times 10^{-4}$ \\
\hline
\end{tabular}




\begin{tabular}{l|cc}
\hline Adaptive NN & $4.2926 \times 10^{-4}$ & $2.1745 \times 10^{-4}$ \\
ANSMC & 0.0082 & 0.0070 \\
ANISMC & $3.9397 \times 10^{-4}$ & $1.9863 \times 10^{-4}$ \\
\hline
\end{tabular}

Table II: Comparison among the controllers in terms of root mean square error (RMSE) for the system with the uncertainty $F_{2}$

\begin{tabular}{l|cc}
\hline \multicolumn{1}{c|}{$R M S E$} & $\left\|E_{x y}\right\|$ & $\left\|E_{\psi}\right\|$ \\
Controller & & \\
\hline SMC & 0.0242 & 0.0351 \\
ABSMC & 0.002 & 0.007 \\
Adaptive NN & 0.0232 & 0.0308 \\
ANSMC & 0.0261 & 0.0369 \\
ANISMC & $3.6058 \times 10^{-4}$ & $3.0998 \times 10^{-4}$ \\
\hline
\end{tabular}

Remark 10: It can be seen from (49) and (50) that the adaptation gain, i.e., $\hat{\kappa}$, must increase to approximate the value $\kappa$ to guarantee the existing condition of sliding mode. The adaptation is stopped and the gains remain fixed once sliding is obtained, i.e., $|\sigma| \leq \varepsilon$. If sliding is then subsequently broken (due to the effects of aggressive disturbance or new disturbance, etc.), adaptation resumes and the gains once more increase. This analysis is used to explain the responses of the adaptive sliding gains (50) shown in Fig. 9. The adaptation gains will be definitely convergent to some certain values when it is big enough to always guarantee the existing condition of sliding mode. The faster of the convergence can be obtained if the value $\varepsilon$ is bigger, and vice versa. For example, in Fig. 11, when the bigger value of $\varepsilon$ is selected, i.e., $\varepsilon=0.05$, the gains are converged quicker compared to Fig. 9 , where the smaller $\varepsilon$ is selected, i.e., $\varepsilon=0.01$.

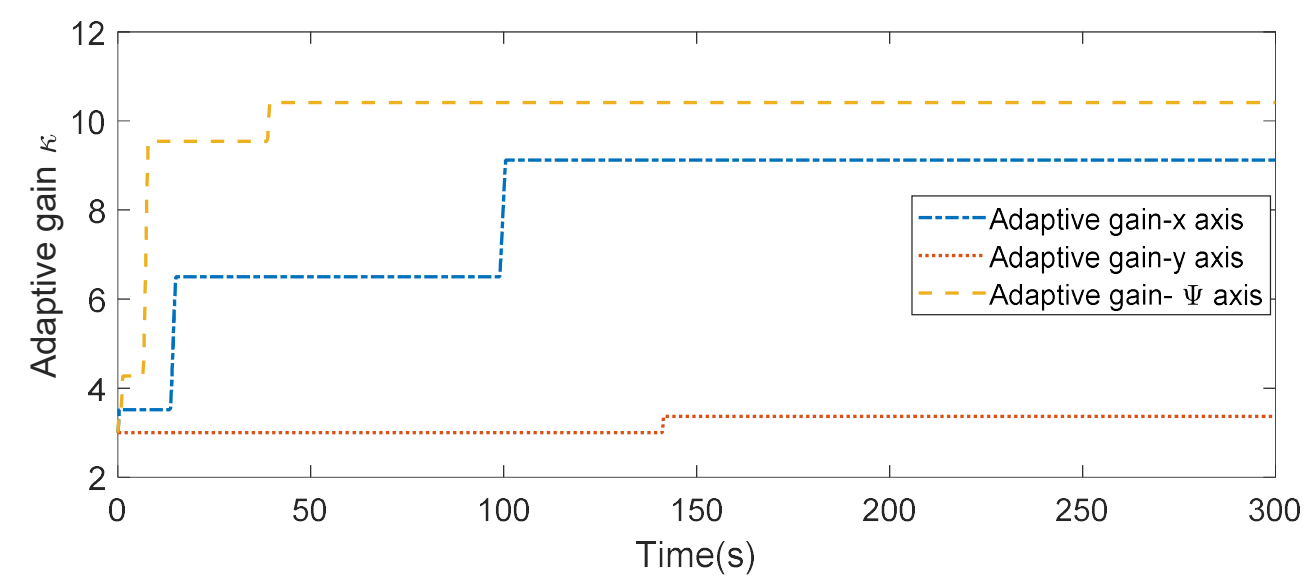


Figure 11. The responses of the adaptive sliding gains in (50) of the proposed ANISMC for the system with the presence of uncertainty $F_{2}$ when $\varepsilon=0.05$.

Remark 11: In the conventional ISMC (8), according to (11), the sliding gain of the switching term is $\Xi^{-1}(\chi+\vartheta)$, where $\|\Psi(\eta, v, t)\| \leq \chi$, whist in the proposed NISMC (9), the sliding gain of the switching term is $\Xi^{-1}(\kappa+\vartheta)$, where $\|\delta\| \leq \kappa$. Due to the approximation capability of NN as stated in Lemma 1, the NN's approximation error, i.e., $\delta$, is usually smaller than the approximation function, i.e., $\Psi(\eta, v, t)$. Consequently, the sliding gain $\kappa$ is smaller than the gain $\chi$. To support this statement, we compare the adaptation gains of the adaptive ISMC controller, which is shown in Fig. 12, and the adaptation gains of the ANISMC controller, which is shown in Fig. 11. From Figs. 11 and 12, it is obvious to see that the adaptation gains of the ANISMC are smaller and convergent faster than that of the ISMC. As a result, we can verify that the ANISMC provides less chattering compared to the ISMC, since the chattering is proportional to the switching gain.

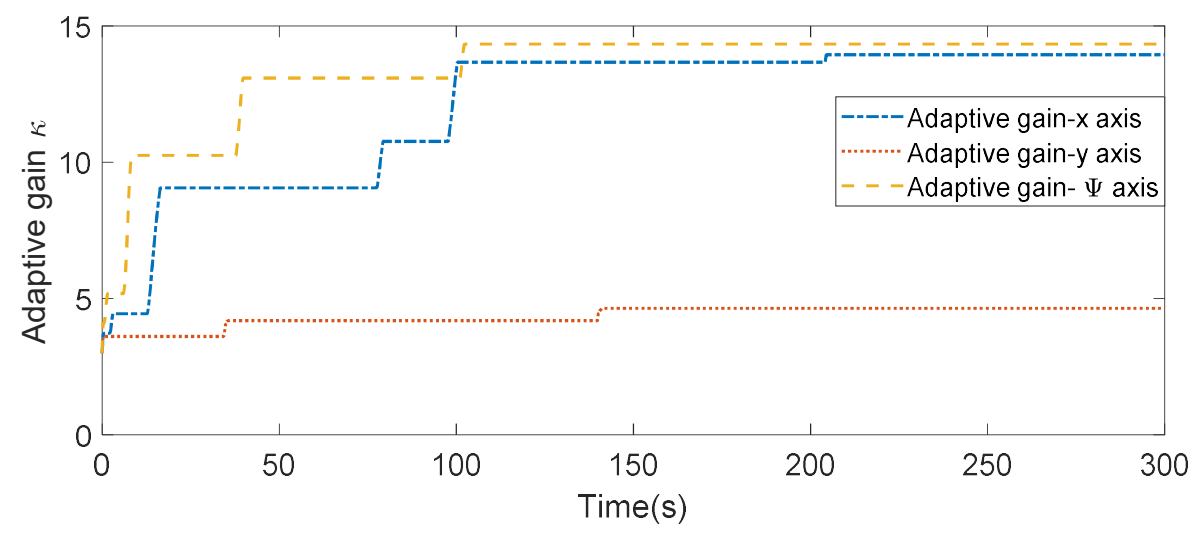

Figure 12. The responses of the adaptive sliding gains of the ISMC for the system with the presence of uncertainty $F_{2}$ when $\varepsilon=0.05$.

Remark 12: In this paper, the effects of the input saturation of the actuator have not been considered during the design of the controller. Thus, the control efforts of the controllers may violate the constraints of the actuator for some circumstances in practical applications. Solving this issue will be investigated in the future works. However, in the literature, several algorithms have been developed to deal with the input saturation issue. Among them, the most popular methods have been described in [47] and [48]. Particularly, in [47], the Nussbaum function has been developed to compensate for the nonlinear term generating by the effects of the input saturation. In [48], the auxiliary design system has been introduced to tackle the saturation effects. The interested readers are 
recommended to refer to [47] and [48] for solving the input saturation issue.

\section{Conclusions}

The paper has been initialized by reviewing the gaps in developing controllers for the tracking control of surface vessels. Then, in order to fill in the gaps and enhance the tracking control for surface vessels, a novel adaptive neural integral sliding mode control has been proposed. By developing a new adaptive integral sliding mode surface, the proposed method preserved many interested features such as eliminating the reaching phase, providing fast transient response, reducing the chattering, and eliminating the requirement on the prior knowledge of the bounded value of the uncertainty and disturbance. The stability of the whole system has been proved thoroughly based on Lyapunov criteria via the backstepping control framework. The comprehensive comparisons with other state-of-the-art control methods have been done for an example of tracking control of surface vessels. The results demonstrated the superior performance of the developed control strategy.

Since this paper focused on developing a novel adaptive neural integral sliding mode surface, the other complex issues for the dynamics model of the system such as input saturation or actuator fault, etc., have not been considered yet. The issues will be studied and investigated in the future works.

\section{Conflict of Interests}

The authors declare that there is no conflict of interests regarding the publication of this paper.

\section{References}

[1] B. Xiao, X. Yang and X. Huo, "A Novel Disturbance Estimation Scheme for Formation Control of Ocean Surface Vessels," IEEE Transactions on Industrial Electronics, vol. 64, no. 6, pp. 4994-5003, June 2017.

[2] S. L. Dai, M. Wang and C. Wang, "Neural Learning Control of Marine Surface Vessels With Guaranteed Transient Tracking Performance," IEEE Transactions on Industrial Electronics, vol. 63, no. 3, pp. 17171727, March 2016.

[3] M. C. Fang, Y. H. Lin, B. J. Wang, "Applying the PD controller on the roll reduction and track keeping for the ship advancing in waves" Ocean Engineering, vol. 54, pp. 13-25, 2012.

[4] M. C. Fang, Y. Z. Zhuo, Z. Y. Lee, "The application of the self-tuning neural network PID controller on the ship roll reduction in random waves," Ocean Engineering, vol. 37, no. 7, pp. 529-538, 2010. 
[5] K. Ishaque, S.S. Abdullah, S.M. Ayob, Z. Salam, "A simplified approach to design fuzzy logic controller for an underwater vehicle," Ocean Engineering, vol. 38, no. 1, pp. 271-284, 2011.

[6] J. Menoyo Larrazabal, M. Santos Peñas, "Intelligent rudder control of an unmanned surface vessel," Expert Systems with Applications, vol. 55, pp. 106-117, 2016.

[7] J. Ghommam, F. Mnif, A. Benali and N. Derbel, "Asymptotic Backstepping Stabilization of an Underactuated Surface Vessel," IEEE Transactions on Control Systems Technology, vol. 14, no. 6, pp. 1150-1157, Nov. 2006.

[8] Z. Yan and J. Wang, "Model Predictive Control for Tracking of Underactuated Vessels Based on Recurrent Neural Networks," IEEE Journal of Oceanic Engineering, vol. 37, no. 4, pp. 717-726, Oct. 2012.

[9] M. Abdelaal, M. Fränzle, A. Hahn, "Nonlinear Model Predictive Control for trajectory tracking and collision avoidance of underactuated vessels with disturbances," Ocean Engineering, vol. 160, pp. 168$180,2018$.

[10] J. Shin, D. J. Kwak and Y. I. Lee, "Adaptive Path-Following Control for an Unmanned Surface Vessel Using an Identified Dynamic Model," IEEE/ASME Transactions on Mechatronics, vol. 22, no. 3, pp. 1143-1153, June 2017.

[11]Z. Yin, W. He, C. Yang, C. Sun, “Control design of marine vessel using reinforcement learning,” Neurocomputing, vol. 311, pp. 353-362, 2018.

[12] Y. Zhang, S. Li and X. Liu, "Adaptive Near-Optimal Control of Uncertain Systems With Application to Underactuated Surface Vessels," IEEE Transactions on Control Systems Technology, vol. 26, no. 4, pp. 1204-1218, July 2018.

[13]B. Xiao, X. Yang and X. Huo, "A Novel Disturbance Estimation Scheme for Formation Control of Ocean Surface Vessels," IEEE Transactions on Industrial Electronics, vol. 64, no. 6, pp. 4994-5003, June 2017.

[14] Jialu Du, Xin Hu, Miroslav Krstić, Yuqing Sun, "Robust dynamic positioning of ships with disturbances under input saturation," Automatica, vol. 73, pp. 207-214, 2016.

[15]Z. Zheng and M. Feroskhan, "Path Following of a Surface Vessel With Prescribed Performance in the Presence of Input Saturation and External Disturbances," IEEE/ASME Transactions on Mechatronics, vol. 22, no. 6, pp. 25642575, Dec. 2017.

[16] M. Mirzaei, N. Meskin and F. Abdollahi, "Robust consensus of autonomous underactuated surface vessels," IET Control Theory \& Applications, vol. 11, no. 4, pp. 486-494, 2017.

[17]V. Utkin, Sliding Modes on Control and Optimization. Berlin, Germany: Springer-Verlag, 1992. 
[18]H. Ashrafiuon, K. R. Muske, L. C. McNinch and R. A. Soltan, "Sliding-Mode Tracking Control of Surface Vessels," IEEE Transactions on Industrial Electronics, vol. 55, no. 11, pp. 4004-4012, Nov. 2008.

[19]R. Yu, Q. Zhu, G. Xia and Z. Liu, "Sliding mode tracking control of an underactuated surface vessel," IET Control Theory \& Applications, vol. 6, no. 3, pp. 461-466, 2012.

[20] S. Yin and B. Xiao, "Tracking Control of Surface Ships With Disturbance and Uncertainties Rejection Capability," IEEE/ASME Transactions on Mechatronics, vol. 22, no. 3, pp. 1154-1162, June 2017.

[21]M. T. Hamayun, C. Edwards and H. Alwi, "Design and Analysis of an Integral Sliding Mode Fault-Tolerant Control Scheme," IEEE Transactions on Automatic Control, vol. 57, no. 7, pp. 1783-1789, July 2012.

[22] Y. Pan, C. Yang, L. Pan and H. Yu, "Integral Sliding Mode Control: Performance, Modification and Improvement," IEEE Transactions on Industrial Informatics, vol. 14, no. 7, 3087-3096, 2018.

[23] Wen-Jun Cao and Jian-Xin Xu, "Nonlinear integral-type sliding surface for both matched and unmatched uncertain systems," IEEE Transactions on Automatic Control, vol. 49, no. 8, pp. 1355-1360, Aug. 2004.

[24] J. Qin, Q. Ma, H. Gao and W. X. Zheng, "Fault-Tolerant Cooperative Tracking Control via Integral Sliding Mode Control Technique," IEEE/ASME Transactions on Mechatronics, vol. 23, no. 1, pp. 342-351, Feb. 2018.

[25]J. Zhang, X. Liu, Y. Xia, Z. Zuo and Y. Wang, "Disturbance Observer-Based Integral Sliding-Mode Control for Systems With Mismatched Disturbances," IEEE Transactions on Industrial Electronics, vol. 63, no. 11, pp. 70407048, Nov. 2016.

[26]H. P. Ren, J. Hu and J. Li, "Conditional integral sliding mode variable structure control for two-tank system," 2017 12th IEEE Conference on Industrial Electronics and Applications (ICIEA), Siem Reap, 2017, pp. 326-331.

[27]H. P. Ren and X. Guo, "Robust Adaptive Control of a CACZVS Three-Phase PFC Converter for Power Supply of Silicon Growth Furnace," IEEE Transactions on Industrial Electronics, vol. 63, no. 2, pp. 903912, Feb. 2016.

[28]H. Khebbache, M. Tadjine, "Robust fuzzy backstepping sliding mode controller for a quadrotor unmanned aerial vehicle," Journal of Control Engineering and Applied Informatics, vol. 15, no. 2, pp. 3-11, 2013.

[29] M. Van, S. S. Ge and H. Ren, "Robust Fault-Tolerant Control for a Class of Second-Order Nonlinear Systems Using an Adaptive Third-Order Sliding Mode Control," IEEE Transactions on Systems, Man, and Cybernetics: Systems, vol. 47, no. 2, pp. 221-228, Feb. 2017. 
[30] S.S. Ge, C.C. Hang, T.H. Hang, T. Zhang, Stable Adaptive Neural Network Control, Kluwer, Boston, MA, USA, 2002.

[31] M. Van, "An Enhanced Robust Fault Tolerant Control Based on an Adaptive Fuzzy PID-Nonsingular Fast Terminal Sliding Mode Control for Uncertain Nonlinear Systems," IEEE/ASME Transactions on Mechatronics, vol. 23, no. 3, pp. 1362-1371, June 2018.

[32]Z. Zhao, W. He and S. S. Ge, "Adaptive Neural Network Control of a Fully Actuated Marine Surface Vessel With Multiple Output Constraints," IEEE Transactions on Control Systems Technology, vol. 22, no. 4, pp. 1536-1543, July 2014.

[33]W. He, Z. Yin and C. Sun, "Adaptive Neural Network Control of a Marine Vessel With Constraints Using the Asymmetric Barrier Lyapunov Function," IEEE Transactions on Cybernetics, vol. 47, no. 7, pp. 1641-1651, July 2017.

[34] L. Liu, Y. J. Liu and S. Tong, "Neural Networks-Based Adaptive Finite-Time Fault-Tolerant Control for a Class of Strict-Feedback Switched Nonlinear Systems," IEEE Transactions on Cybernetics. doi: 10.1109/TCYB.2018.2828308.

[35] Y. Li, S. Tong, L. Liu, G. Feng, "Adaptive output feedback control design with prescribed performance for switched nonlinear systems," Automatica, vol. 80, pp. 225-231, 2017.

[36] A. M. Zou, K. D. Kumar, Z. G. Hou and X. Liu, "Finite-Time Attitude Tracking Control for Spacecraft Using Terminal Sliding Mode and Chebyshev Neural Network," IEEE Transactions on Systems, Man, and Cybernetics, Part B (Cybernetics), vol. 41, no. 4, pp. 950-963, Aug. 2011.

[37] L. Wang, T. Chai and L. Zhai, "Neural-Network-Based Terminal Sliding-Mode Control of Robotic Manipulators Including Actuator Dynamics," IEEE Transactions on Industrial Electronics, vol. 56, no. 9, pp. 3296-3304, Sept. 2009.

[38] Y. Chu, J. Fei, and S. Hou, " Dynamic global proportional integral derivative sliding mode control using radial basis function neural compensator for three-phase active power filter," Transactions of the Institute of Measurement and Control, to be published, doi: https://doi.org/10.1177/0142331217726955.

[39]J. Fei and C. Lu, "Adaptive Sliding Mode Control of Dynamic Systems Using Double Loop Recurrent Neural Network Structure," IEEE Transactions on Neural Networks and Learning Systems, vol. 29, no. 4, pp. 1275 1286, April 2018. 
[40]H. R. Rodriguez, V. P. V. A. Sanchez-Orta, O. Garcia-Salazar, "Robust backstepping control based on integral sliding modes for tracking of quadrators, "J. Intel. Robot. Systems, vol. 73, pp. 51-66, 2014.

[41]W. Haitao, D. Dinmin, X. Jianping, L. Jiaolong, "Dynamic modeling of a hose-drogue aerial refueling system and integral sliding mode backstepping control for the hose whipping phenomenon," Chinese Journal of Aeronautics, vol. 27, no. 4, pp. 930-946, 2014.

[42] M. Van, M. Mavrovouniotis and S. S. Ge, "An Adaptive Backstepping Nonsingular Fast Terminal Sliding Mode Control for Robust Fault Tolerant Control of Robot Manipulators," IEEE Transactions on Systems, Man, and Cybernetics: Systems. doi: 10.1109/TSMC.2017.2782246, to be published, 2018.

[43]C. Peng, Y. Bai, X. Gong, Q. Gao, C. Zhao and Y. Tian, "Modeling and robust backstepping sliding mode control with Adaptive RBFNN for a novel coaxial eight-rotor UAV," IEEE/CAA Journal of Automatica Sinica, vol. 2, no. 1, pp. 56-64, January 102015.

[44]F. J. Lin, S. Y. lee, P. H. Chou, "Intelligent integral backstepping sliding mode control using recurrent neural network for piezo-flexural nanopositioning stage," Asian Journal of Control, vol. 52, no. 2, pp. 456-472, 2016.

[45]F. Plestan, Y. Shtessel, V. Bregeault, A. Poznyak, "New methodologies for adaptive sliding mode control," International Journal of Control, vol. 83, no. 9, pp. 1907-1919, 2009.

[46]Y. Shtessel, M. Taleb, F. Plestan, “A novel adaptive-gain supertwisting sliding mode controller: Methodology and application," Automatica, vol. 48, pp. 759-769, 2012.

[47]C. Wen, J. Zhou, Z. Liu and H. Su, "Robust Adaptive Control of Uncertain Nonlinear Systems in the Presence of Input Saturation and External Disturbance," IEEE Transactions on Automatic Control, vol. 56, no. 7, pp. 1672-1678, July 2011.

[48]M. Chen, S. S. Ge, B. Ren, “Adaptive tracking control of uncertain MIMO nonlinear systems with input constraints" Automatica, vol. 47, no. 3, pp. 452-465, 2011. 